\title{
The complete census of optically selected AGNs in the Coma supercluster: the dependence of AGN activity on the local environment ${ }^{\star}$
}

\author{
G. Gavazzi ${ }^{1}$, G. Savorgnan ${ }^{1}$, and Mattia Fumagalli ${ }^{2}$ \\ 1 Dipartimento di Fisica G. Occhialini, Università di Milano-Bicocca, Piazza della Scienza 3, 20126 Milano, Italy \\ e-mail: giuseppe.gavazzi@mib.infn.it \\ 2 Leiden Observatory, Leiden University, PO Box 9513, 2300 RA Leiden, The Netherlands \\ e-mail: fumagalli@strw.leidenuniv.nl
}

Received 10 June 2011 / Accepted 18 July 2011

\section{ABSTRACT}

\begin{abstract}
Aims. To investigate the dependence of the occurrence of active galactic nuclei (AGNs) on local galaxy density, we study the nuclear properties of $\sim 5000$ galaxies in the Coma supercluster whose density spans two orders of magnitude from the sparse filaments to the cores of rich clusters.

Methods. We obtained optical spectra of the nuclei of 283 galaxies using the $1.5 \mathrm{~m}$ Cassini telescope of Bologna observatory. Among these galaxies, 177 belong to the Coma supercluster and are added to the 4785 spectra available from SDSS (DR7) to fill-in the incomplete coverage by SDSS of luminous galaxies. We perform a spectral classification of the nuclei of galaxies in this region (with a completeness of $98 \%$ at $r \leq 17.77$ ), classifying the nuclear spectra in six classes: three of them (SEY, sAGN, LIN) refer to AGNs and the remaining three (HII, RET, PAS) refer to different stages of starburst activity. We perform this classification as recommended by Cid Fernandes and collaborators using the ratio of $\lambda 6584$ [NII] to $\mathrm{H} \alpha$ lines and the equivalent width of $\mathrm{H} \alpha$ (WHAN diagnostic), after correcting the last quantity by $1.3 \AA$ for underlying absorption.

Results. We find that $482(10 \%)$ of 5027 galaxies host an AGN: their frequency strongly increases with increasing luminosity of the parent galaxies, such that $32 \%$ of galaxies with $\log \left(L_{i} / L_{\odot}\right) \geq 10.2$ harbor an AGN at their interior. In addition to their presence in luminous galaxies, AGNs are also found in red galaxies with $\langle g-i\rangle \simeq 1.15 \pm 0.15$ mag. The majority of SEY and sAGN (strong AGNs) are associated with luminous late-type (or S0a) galaxies, while LIN (weak AGNs) and RET ("retired": nuclei that have experienced a starburst phase in the past and are now ionized by their hot evolved low-mass stars), are mostly found among E/SOas. The number density of AGNs, HII region-like, and retired galaxies is found to anti-correlate with the local density of galaxies, such that their frequency drops by a factor of two near the cluster cores, while the frequency of galaxies containing passive nuclei increases by the same amount towards the center of rich clusters. The dependence of AGN number density on the local galaxy density is greater than the one implied by morphology segregation alone.
\end{abstract}

Key words. galaxies: clusters: individual: Coma - galaxies: evolution - galaxies: nuclei

\section{Introduction}

It appears that $1.5 \mathrm{~m}$ class telescopes, especially those ones located in zones of marginal climatic conditions, have lost much of their raison d'etre, particularly after the advent of the Sloan Digital Sky Survey (SDSS). They however maintain a crucial didactic value for the training of young astronomers and provided that they are employed for suitable projects, can still make some significant niche contribution. One appropriate project in this respect is, in our opinion, the determination of the spectral properties of nuclei of bright galaxies, as part of a general study to perform a complete census of active galactic nuclei (AGNs). A spectral resolution on the order of $R=1000$ in the red channel (the blue-arm is more penalized by the relative blindness of CCDs) is sufficient to measure in a matter of a few minutes, even in non-photometric conditions, some crucial line ratios in the nuclear regions of galaxies, owing to their high surface brightness.

In this spirit, one of us (G.G.) invited yearly his first year master students to participate in some observing runs at the $1.5 \mathrm{~m}$ Loiano telescope of the Observatory of Bologna (It).

* Table 4 is available in electronic form at http: //www . aanda.org
Unsurprisingly, many nights were affected by poor weather, although an amazing wealth of nuclear spectra (283) of nearby galaxies was accumulated, suitable for detecting any AGNs harbored in their interior.

A large amount of data (177) were gathered for the region of the Coma supercluster, on which this work is focused. Following a fortunate run of 7/8 photometric nights in spring 2011 and the appearance of Mahajan et al. (2010), who investigated the AGN environmental dependence in the Coma supercluster relying purely on SDSS data, regardless of its well-known incompleteness at the bright end (Blanton et al. 2005a,b,c), we decided to publish our measurements derived in the past six years, with the hope that they might contribute to improving our understanding of AGNs.

There is a clear need to establish the frequency of AGNs of various types, in various environments, locally and in a cosmological perspective, to improve our understanding of galaxy assembly. Nevertheless, even after the advent of SDSS, which has permitted many extensive studies of galaxies, the processes involved remain unclear. Hao et al. (2005) estimated that $4-10 \%$ of all galaxies in the SDSS harbor an AGN; 
Kauffmann et al. (2003) estimated that up to $80 \%$ of galaxies more massive than $10^{11} M_{\odot}$ host a supermassive black hole, either dormant or active. The role of the environment in triggering or inhibiting nuclear activity remains disputed. Kauffmann et al. (2004), analyzing 122000 SDSS spectra, found a decrease in the fraction of powerful AGNs (with high [OIII] luminosity, i.e. dominated by Seyferts) with increasing galaxy environment density, mainly because the most powerful AGNs are hosted by late-type galaxies, which are the least likely to exist in dense environments. Popesso \& Biviano (2006) found that the AGN fraction decreases with the increasing velocity dispersion of galaxies in groups and clusters, being higher in dense, low-dispersion groups, in contrast to the findings of Shen et al. (2007) \& Miller et al. (2003) who found that the frequency of low activity AGNs does not correlate with environment.

The classification of AGNs based on optical spectra has been traditionally performed using the BPT diagnostic diagram of Baldwin et al. (1981), which adopts the measurement of at least four spectral lines: $\mathrm{H} \beta, \mathrm{O}[\mathrm{III}], \mathrm{H} \alpha$, and $\mathrm{N}[\mathrm{II}]$. The ratio $\mathrm{H} \alpha / \mathrm{N}[\mathrm{II}]$ (where $\mathrm{H} \alpha$ must be corrected for any underlying stellar absorption, as stressed by Ho et al. 1997) differentiates AGNs from HII region-like nuclei, while the ratio $\mathrm{H} \beta / \mathrm{O}[\mathrm{III}]$ allows us to separate the strong AGNs from the weaker LINERs. More recently Cid Fernandes et al. $(2010,2011)$ introduced a two-line diagnostic diagram, named WHAN, based on the $\mathrm{H} \alpha / \mathrm{N}[\mathrm{II}]$ ratio combined with the strength of the $\mathrm{H} \alpha$ line (corrected for underlying stellar absorption) to discriminate both strong and weak AGNs, which are both supposed to be ionized by active black holes, from "fake AGNs", dubbed "retired galaxies", whose ionization mechanism is provided by their old stellar population. As argued by several authors (e.g. Trinchieri \& di Serego Alighieri 1991; Binette et al. 1994; Macchetto et al. 1996; Stasińska et al. 2008; Sarzi et al. 2010; Capetti \& Baldi 2011), hot evolved stars, such as postasymptotic giant branch stars and white dwarfs, can produce a substantial diffuse field of ionizing photons: it has been shown that they can produce emission lines with ratios mimicking those of AGNs and - in particular - of LINERs.

An equivalent width (EW) of $\mathrm{H} \alpha$ of at least $3 \AA$ is ultimately required for a nucleus to be considered ionized by a central black hole (Cid Fernandes et al. 2010, 2011). This quantitative threshold of course might be questioned. Among the galaxies in our sample, we might consider for example the elliptical NGC 3862 (CGCG 97-127), which is the second brightest galaxy of A1367 and was classified as an AGN by Véron-Cetty \& Véron (2006). It harbors the radio galaxy 3C-264 (Gavazzi et al. 1981) and an optical jet (Crane et al. 1993), leaving little doubt that it contains a central monster. Its optical spectrum (taken either at Loiano or from SDSS) displays a strong NII emission line (of EW $4.81 \AA$ ) and a weak $\mathrm{H} \alpha \mathrm{EW}$ of $1.51 \AA$. After adding $1.3 \AA$ to the underlying absorption, the galaxy just misses the $\mathrm{H} \alpha>3 \AA$ threshold, thereby fails to be classified as a LIN but not as a RET according to the criteria of Cid Fernandes et al. $(2010,2011)^{1}$.

The Coma supercluster, on which this paper is focused, is an ideal laboratory for initiating a complete census of AGNs at $z=0$ because problems related to the incompleteness of SDSS are less severe here than elsewhere. Even its largest sized galaxies have diameters $<2-3$ arcmin, making the "shredding" problem much less severe than for other nearby environments (e.g. Virgo). The brightest galaxies have been well-studied and even the faintest galaxies belonging to the Coma supercluster have sizes in excess of 10 arcsec, allowing their classification

\footnotetext{
${ }^{1}$ Similar examples (see Sect. 4) convinced us to lower the threshold for LIN from $3 \AA$ to $1.5 \AA$ in the present analysis.
}

by visual inspection on SDSS plates. Moreover, at the distance of $100 \mathrm{Mpc}$ in the Coma supercluster, the three arcsec fibers adopted by SDSS provide truly "nuclear" spectra. Studying the Coma supercluster using both the integrated and nuclear properties of galaxies at $z=0$ derived from SDSS data, allows us to compare galaxies properties for a variety of environmental conditions, from the center of two rich clusters to the sparse filamentary regions where the local galaxy density is $\sim 100$ times lower, without the biases caused by studying galaxies at different distances. It provides a complementary view to the many statistical analyses that have been made using SDSS data (e.g. Kauffmann et al. 2004). Combined with studies of galaxy evolution at higher redshift it will shed light on the mechanisms and processes that contribute to the evolution of galaxies in the cosmological context.

Owing to the two-line WHAN diagnostics, the red-channel spectra taken at Loiano can contribute significantly to the census of AGNs by filling the residual incompleteness of the SDSS spectral database for luminous galaxies caused by shredding and fiber conflict (Blanton et al. 2005a,b,c).

The layout of this paper is as follows. In Sect. 3, we illustrate the observations taken at the Loiano observatory and the data reduction procedures. The nuclear spectra are given and classified in Sect. 4. The census of AGNs of the various types in the Coma supercluster is carried out in Sect. 5. Our results are compared with Mahajan et al. (2010) and discussed in Sect. 6. The standard cosmology is assumed, with $H_{0}=73 \mathrm{~km} \mathrm{~s}^{-1} \mathrm{Mpc}^{-1}$.

\section{The sample}

This paper is based on two samples:

i) a miscellaneous set of 283 relatively bright galaxies, not complete by any means, for which we took long-slit, red-channel nuclear spectra of $R \sim 1000$, with the Loiano telescope. We refer to this as the "LOI sample" hereafter;

ii) a complete set of 5027 galaxies (hereafter the "COMA sample") selected in the region $11.5^{\mathrm{h}}<\mathrm{RA}<13.5^{\mathrm{h}} ;+18^{\circ}<$ Dec $<32^{\circ}$ (see GoldMine, Gavazzi et al. 2003) that traditionally describes the Coma supercluster and was extensively studied by Gavazzi et al. (2010), combined with an extended region defined by $10^{\mathrm{h}}<\mathrm{RA}<16^{\mathrm{h}}$ and $+24^{\circ}<$ Dec $<28^{\circ}$ (see Fig. 1). The second area is suitable for filling an LST interval corresponding to the duration of an observing night in spring and has also been covered with HI observations by ALFALFA (Giovanelli et al. 2005), whose HI selected targets will shortly be publicly available.

Following the method of Gavazzi et al. (2010), we searched in this area of the sky $\left(11.5^{\mathrm{h}}<\mathrm{RA}<13.5^{\mathrm{h}} ;+18^{\circ}<\right.$ Dec $<32^{\circ}$ combined with $10^{\mathrm{h}}<\mathrm{RA}<16^{\mathrm{h}} ;+24^{\circ}<$ Dec $\left.<28^{\circ}\right)$ the SDSS DR7 spectroscopic database (Abazajian et al. 2009) for all galaxies with $r \leq 17.77$ in the redshift interval $3900<c z<$ $9500 \mathrm{~km} \mathrm{~s}^{-1}$. We obtained 4790 targets. For each, we extracted the coordinates, $u, g, r, i, z$ Petrosian magnitudes (AB system), and spectroscopic information, including the principal line intensities and the redshift. The morphological classification of all galaxies was performed by individual visual inspection of SDSS color images.

To fill-in the incompleteness of SDSS for luminous galaxies due to shredding and fiber conflict (Blanton et al. 2005a,b,c), we added 133 CGCG (Zwicky et al. 1961-1968) galaxies with known redshifts from NED that were not included in the SDSS spectral database (see Fig. 2). For these objects, we also 

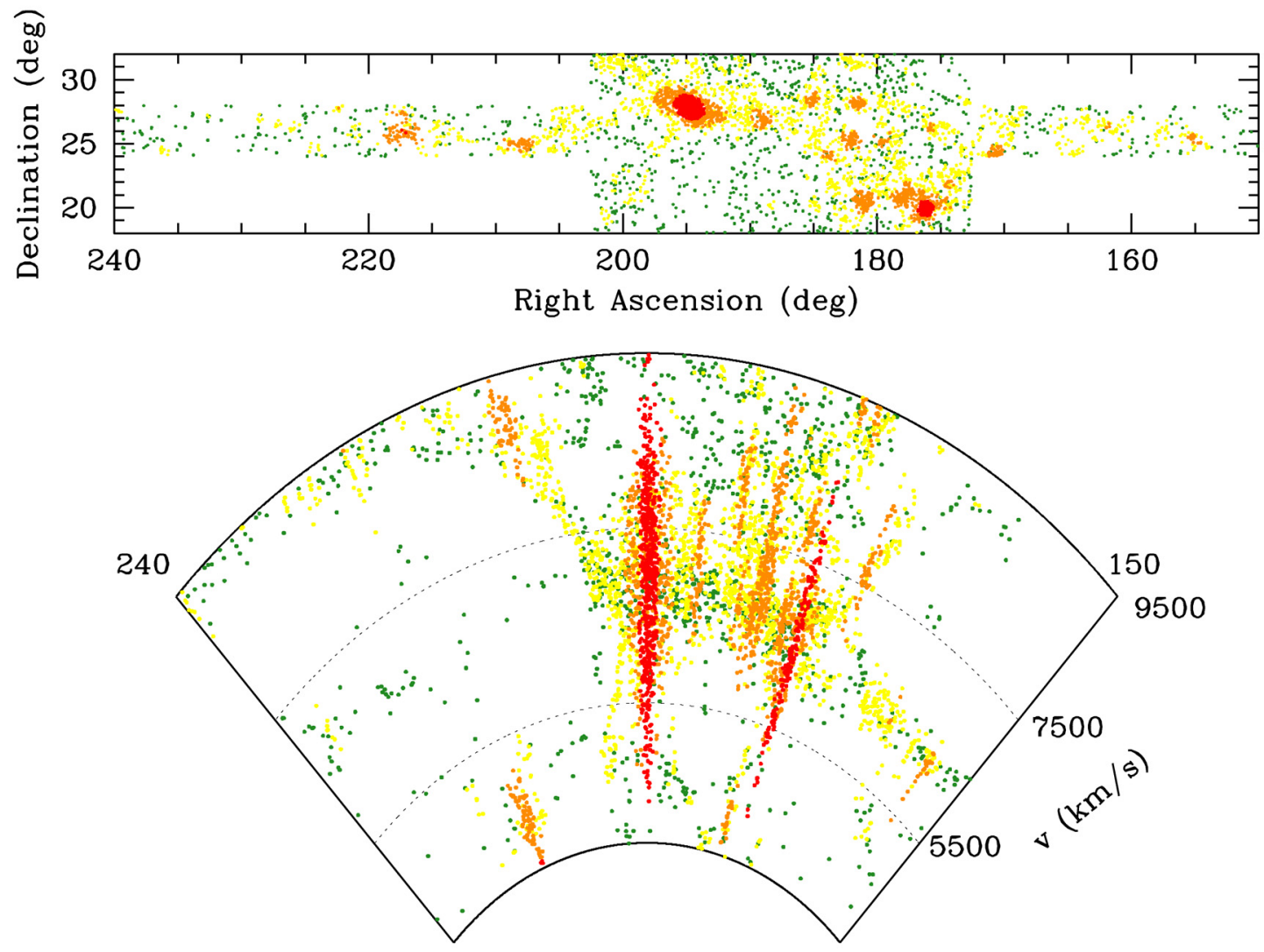

Fig. 1. (Top panel) The Coma supercluster region studied in this work in celestial coordinates and as a wedge-diagram. (Bottom panel) Symbols are color coded according to the density parameter (see Sect. 5): UL: $\delta_{1,1000} \leq 0$ are green; L: $0<\delta_{1,1000} \leq 4$ are yellow; H: $4<\delta_{1,1000} \leq 20$ are orange; UH: $\delta_{1,1000}>20$ are red.

measured the $u, g, r, i, z$ Petrosian magnitudes using the SDSS DR7 navigation tool, which provides accurate magnitudes.

Additional galaxies that could not be found in the SDSS spectroscopic catalog were searched for using NED. For these targets, we again evaluated the $u, g, r, i, z$ magnitudes using the SDSS navigator tool and among them we selected 76 objects meeting the condition $r \leq 17.77$, which matches the selection criterion of the SDSS spectral catalog (Strauss et al. 2002). We repeated a similar search in the ALFALFA database (Haynes, private communication) for the region $10^{\mathrm{h}}<\mathrm{RA}<16^{\mathrm{h}}$ and $+24^{\circ}<$ Dec $<28^{\circ}$, and found 28 additional HI-selected systems with $r \leq 17.77$, not included in the SDSS spectral database. In total, our sample consists of 5027 galaxies: 4790 from SDSS and 237 from other sources.

The 4785 out of 4790 galaxies selected from SDSS have a spectrum available from the SDSS database (five remaining objects have partly corrupted spectra); another 130 were observed at Loiano, taken from sample i), and the remaining 112 do not yet have available spectra. In total, the "COMA sample" reaches a spectral completeness of $98 \%$.

\section{Observations and data reduction}

Optical spectra of the nuclei of 283 galaxies in sample i) were obtained during 14 runs from 2005 to 2011 (see Table 1) using the Bologna Faint Object Spectrograph and Camera (BFOSC) attached to the $152 \mathrm{~cm} \mathrm{~F} / 8$ Cassini Telescope located in Loiano, belonging to the Observatory of Bologna. These consist of long-slit spectra taken through a two-arcsec slit, combined with

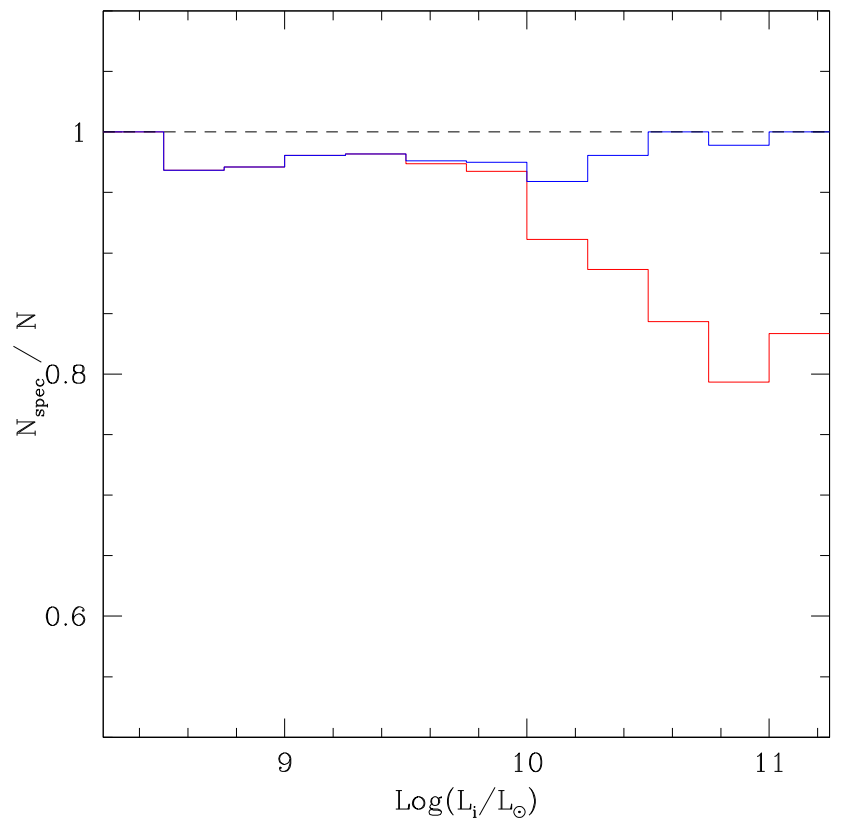

Fig. 2. The completeness of the "COMA sample", consisting of galaxies taken from SDSS, targets taken from CGCG, NED, and ALFALFA, and objects with spectra taken at Loiano (blue), is $98 \%$ (only 112 galaxies remaining without nuclear spectra). Galaxies selected from the SDSS alone (red), e.g. used by Mahajan et al. (2010) are incomplete by $20 \%$ in the highest luminosity bins, where most AGNs are expected. 
Table 1. Log of the observations at Loiano.

\begin{tabular}{llll}
\hline \hline Year & Feb. & Mar. & $N_{\text {spec }}$ \\
\hline 2005 & $9,10,11$ & $9,11,12$ & 45 \\
2006 & 26 & 23 & 24 \\
2007 & $13,15,16$ & 17,18 & 29 \\
2008 & 5 & 3 & 10 \\
2009 & $18,19,21$ & 26,27 & 54 \\
2010 & 8 & - & 10 \\
2011 & $7,8,9,10$ & $6,7,8$ & 133 \\
\hline
\end{tabular}

Notes. Two new-moon periods of four nights each were allocated to the present project per year. We list information for the useful nights. The total number of spectra (305) includes 22 of objects that were repeatedly observed during several runs. In these cases, Table 2 describes the combined spectra.

an intermediate-resolution grism $(R \sim 1000)$ covering the 6100-8200 $\AA$ portion of the red-channel and containing $\mathrm{H} \alpha$, [NII], and [SII] spectral lines. BFOSC is equipped with a EEV LN/1300-EB/1 CCD detector of $1300 \times 1340$ pixels, reaching $90 \%$ QE near $5500 \AA$. For the spatial scale of $0.58 \mathrm{arcsec} /$ pixel, the useful field is of $12.6 \times 13$ square arcmin. The grism dispersion of $8.8 \mathrm{~nm} / \mathrm{mm}$ results in spectra with $1.6 \AA /$ pix. Exposures of 3-5 min were repeated typically three times (to help remove the cosmic ray hits), up to six times. The slit was generally set in the E-W direction, except when aligned along the galaxy major axis, or along the direction connecting two nearby objects taken simultaneously in the slit. The wavelength calibration was secured by means of frequent exposures of a $\mathrm{He}-\mathrm{Ar}$ hollow-cathode lamp. The flux calibration and the spectrograph response were obtained by daily exposures of the star Feige34. The typical seeing conditions at Loiano ranged from 1.5 to 2.5 arcsec.

The reduction of the spectra was based on the IRAF STSDAS $^{2}$ reduction packages. The wavelength calibration was performed on the lamp exposures using identify-reidentifyfitcoo and transferred to the scientific exposures using transform. After checking the wavelength calibration using the principal sky lines, these were removed using background. One-dimensional (1d) spectra of the nuclei were extracted from the two-dimensional (2d) images using apsum in apertures of 5.8 arcsec. After flux calibration, the $1 \mathrm{~d}$ spectra were normalized to the intensity of the continuum under $\mathrm{H} \alpha$, transformed to rest-frame velocity, and measured with splot to obtain the EW of the $\mathrm{H} \alpha$ and $\lambda 6584$ [NII] lines.

\section{Spectral classification}

The classification of the nuclear activity based on optical spectra was performed according to identical rules for sample i) LOI and ii) COMA (except for Seyfert galaxies; see below in this section). The classification rule is based on the WHAN diagram introduced by Cid Fernandes et al. (2010, 2011), which is based on the strength of the $\mathrm{H} \alpha$ line corrected for underlying stellar absorption (Ho et al. 1997) and the ratio of [NII] to $\mathrm{H} \alpha_{\text {corr }}(\mathrm{EW})$ to discriminate between different levels of nuclear activity. As for

\footnotetext{
${ }^{2}$ IRAF is the Image Analysis and Reduction Facility made available to the astronomical community by the National Optical Astronomy Observatories, which are operated by AURA, Inc., under contract with the US National Science Foundation. STSDAS is distributed by the Space Telescope Science Institute, which is operated by the Association of Universities for Research in Astronomy (AURA), Inc., under NASA contract NAS 5-26555.
}

the underlying absorption at $\mathrm{H} \alpha$, Decarli et al. (2007) adopted an average correction of $1.7 \AA$. Owing to the limitations of the SDSS spectral database, this quantity is here determined again to greater accuracy using 881 SDSS spectra of passive galaxies in the Coma supercluster (see Table 3). The mean underlying stellar absorption at $\mathrm{H} \alpha$ present in all passive galaxy spectra amounts to $1.3 \AA$, irrespective of luminosity, below or above $\log \left(L_{i} / L_{\odot}\right)=10^{3}$. Thus we adopt $1.3 \AA$ for the underlying continuum absorption at $\mathrm{H} \alpha$, i.e. $\mathrm{H} \alpha_{\text {corr }}=\mathrm{H} \alpha-1.3 \AA$.

Figure 3 shows the WHAN diagram with the adopted spectral classification thresholds. The vertical line shows the separation between HII region-like nuclei and AGNs. This threshold is set at $\log \left(\mathrm{EW}[\mathrm{NII}] / \mathrm{EWH} \alpha_{\text {corr }}\right)=-0.30$ in agreement with Capetti \& Baldi (2011), as a compromise between the value of -0.22 adopted by Baldwin et al. (1981) and Decarli et al. (2007), and the value of -0.40 adopted by Cid Fernandes et al. (2010, $2011)^{4}$.

In Fig. 3, several EWH $\alpha_{\text {corr }}$ thresholds are indicated. These are used to discriminate between the various levels of nuclear activity, as recommended by Cid Fernandes et al. (2010, 2011) with one difference: the separation between both HII regions and weak AGNs (LIN) from "fake ones" (RET) is set to $\left|\mathrm{EWH} \alpha_{\text {corr }}\right|=1.5 \AA$ instead of $3 \AA^{5}$. Summarizing, we define six independent classes of nuclear activity:

- "HII region-like" nuclei are characterized by intense starformation activity: their spectra display both $\mathrm{H} \alpha$ and [NII] in emission, with $\left|\mathrm{EWH} \alpha_{\text {corr }}\right|>1.5 \AA$ and the ratio $\log \left(\mathrm{EW}[\mathrm{NII}] / \mathrm{EWH} \alpha_{\text {corr }}\right)<-0.3$;

- strong AGN (sAGN) contain a powerful active nucleus (possibly triggered by a supermassive black hole): their spectra have $\left|\mathrm{EWH} \alpha_{\text {corr }}\right|>6 \AA$ and $\log \left(\mathrm{EW}[\mathrm{NII}] / \mathrm{EWH} \alpha_{\text {corr }}\right)>$ -0.3 ;

- weak AGNs (LIN) show the signature of a less active nucleus than sAGN: their spectra have $1.5<\left|\mathrm{EWH} \alpha_{\text {corr }}\right|<6 \AA$ and $\log \left(\mathrm{EW}[\mathrm{NII}] / \mathrm{EWH} \alpha_{\text {corr }}\right)>-0.3$;

- fake AGNs (RET) are thought to be "retired nuclei" whose ionization mechanism is provided by their old stellar

${ }^{3}$ For star-forming galaxies, the direct determination of absorption is hampered by the presence of $\mathrm{H} \alpha$ and $[\mathrm{NII}]$ in emission. However, for bright spirals $\left(\log \left(L_{i} / L_{\odot}\right)>10\right.$, often dominated by bulges) the presence of underlying absorption at $\mathrm{H} \alpha$ is sometimes detected (although difficult to quantify) in the SDSS spectra. In contrast, for faint late-types $\left(\log \left(L_{i} / L_{\odot}\right) \leq 10\right)$ the absorption component at $\mathrm{H} \alpha$ is generally not detected, being overtaken by the emission component, which amounts on average to $30 \AA$ (and exceeds $5 \AA$ for $93 \%$ of them), making the $1.3 \AA$ correction negligible.

4 With this choice, two galaxies (NGC 3773 and CGCG136-042) classified as HII regions in NED are inconsistently classified as LINER by ourselves because they have $-0.3<\log \left(\mathrm{EW}[\mathrm{NII}] / \mathrm{EWH} \alpha_{\text {corr }}\right)<-0.22$, but another two (NGC 2537 and CGCG 71068), classified as HII regions by both NED and us, would have been inconsistently classified as LINERS by Cid Fernandes et al. (2010, 2011).

5 This choice is adopted because well-established LINERS e.g. VCC1110 (Véron-Cetty \& Véron 2006), VCC2070 (Panessa \& Bassani 2002), NGC 2841 (Véron-Cetty \& Véron 2006), NGC 6166 (containing the radio galaxy 3C-338, Gavazzi \& Perola 1980), CGCG 97127 (Véron-Cetty \& Véron 2006; containing the radio galaxy 3C-264, Gavazzi et al. 1981; with optical jet Crane et al. 1993), which would otherwise have been classified as RET by Cid Fernandes et al. (2010, 2011), are consistently classed among LIN. Even after weakening the criterion for LIN to $\mid \mathrm{EWH} \alpha_{\text {corr }}>1.5 \AA$, some inconsistencies remain between our classification and NED: NGC 2685 (classified as Sey2 by Véron-Cetty \& Véron 2006) and VCC1615 (classified as LIN by Véron-Cetty \& Véron 2006) are classified as RET in this work. 


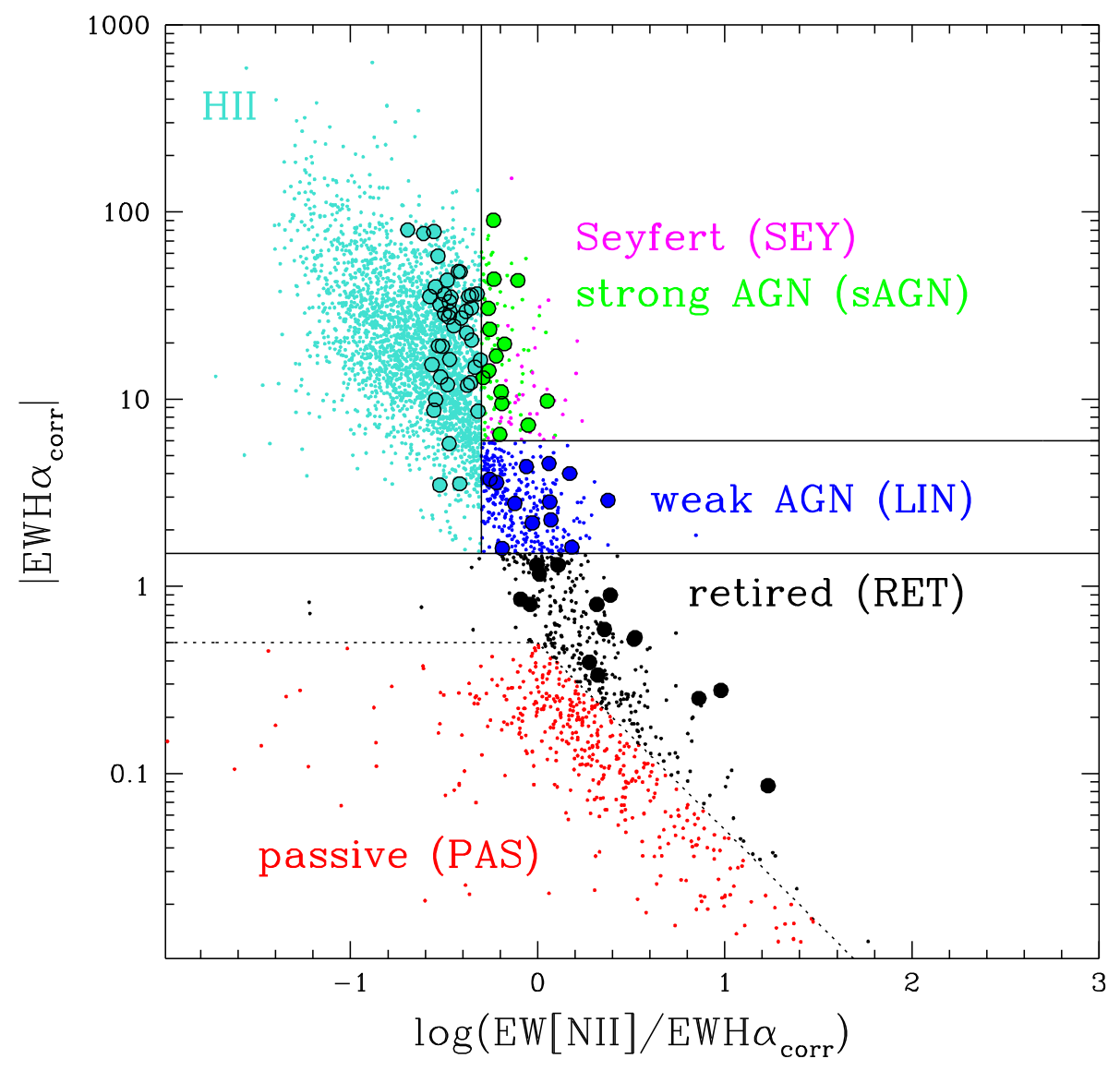

Fig. 3. The WHAN diagnostic diagram. The equivalent width of $\mathrm{H} \alpha$ is corrected by $1.3 \AA$ for underlying absorption. Small symbols refer to the COMA sample, large symbols to the LOI sample. Seyfert 1 galaxies, identified visually in the Loiano spectra, are given as triangles.
Table 2. Line measurements performed on stacked Loiano spectra of $N$ objects in six spectral classes in the "LOI sample".

\begin{tabular}{lccc}
\hline \hline Class & $N$ & EWH $\alpha$ & EW[NII2] \\
\hline HII & 106 & -25.4 & -9 \\
SY1 & 10 & -30 & -24 \\
SAGN & 41 & -13.6 & -9.1 \\
LIN & 42 & -1.7 & -3.0 \\
RET & 12 & 1.0 & -1.1 \\
PAS & 42 & 1.6 & 0.0 \\
\hline
\end{tabular}

Notes. Negative $=$ emission .

population in a way that can mimic the processes of AGNs and - in particular - of LINERs: their spectra have either $\mathrm{H} \alpha$ in moderate absorption $\left(0.5<\left|\mathrm{EWH} \alpha_{\text {corr }}\right|<1.5 \AA\right)$ or both $\mathrm{H} \alpha$ in absorption $\left(\left|\mathrm{EWH} \alpha_{\text {corr }}\right|<0.5 \AA\right)$ and [NII] in emission $(|\mathrm{EW}[\mathrm{NII}]|>0.5 \AA)$;

- passive nuclei (PAS) are typically red, contain exclusively old stars, with no star formation activity: their spectra show both $\mathrm{H} \alpha$ and $[\mathrm{NII}]$ in absorption $\left(\left|\mathrm{EWH} \alpha_{\text {corr }}\right|<0.5 \AA\right.$ and $|\mathrm{EW}[\mathrm{NII}]|<0.5 \AA$ ).

The classification of Seyfert galaxies follows different rules for the "LOI sample" and SDSS spectra. For SDSS spectra, we use EW[OIII]/EWH $\beta>3$ to identify Seyfert (SEY) among sAGN. In the "LOI sample", the absence of blue-channel spectra prevents us from using the BPT diagnostic diagram, hence Seyfert1 galaxies (SY1, represented with pink triangles in Fig. 3) are classified by visual inspection of individual spectra for the presence of broad permitted lines. Tables 2 and 3 summarize the classification of spectra, respectively, in the "LOI" and "COMA" samples.
Table 3. Line statistics in the "COMA sample" containing $N$ objects in each spectral class.

\begin{tabular}{lccc}
\hline \hline Class & $N$ & EWH $\alpha$ & EW[NII2] \\
\hline HII & 2721 & -28.7 & -5.9 \\
SEY & 48 & -13.4 & -12.6 \\
SAGN & 103 & -22.8 & -14.8 \\
LIN & 331 & -1.7 & -2.5 \\
RET & 436 & 1.0 & -1.0 \\
PAS & 881 & 1.3 & -0.2 \\
\hline
\end{tabular}

Notes. Negative $=$ emission.

Template spectra of the six spectral classes, obtained by stacking $\mathrm{N}$ spectra taken at Loiano, are given in Fig. 4 (LOI top panel). The 4785 FITS spectra available from SDSS were downloaded, shifted to their rest-frame using IRAFdopcor, and normalized to the flux intensity near $\mathrm{H} \alpha$. The rms of their continuum were measured between $6230 \AA$ and $6490 \AA$ (avoiding bright galaxy spectral lines and sky residuals), providing an estimate of the signal-to-noise ratio $(S / N)$. Spectra with $S / N>10$ were stacked in each spectral class and are shown in Fig. 4 (COMA bottom panel).

The parameters of the 283 individual spectra for the "LOI sample" are listed in Table 4 as follows: Col. 1: Galaxy name; Cols. 2 and 3: J2000 celestial coordinates; Col. 4: redshift from NED; Cols. 5 and 6: measured EW of $\mathrm{H} \alpha$ and $\lambda 6584$ [NII] lines from the present work (negative values represent emission); Col. 7: the nuclear activity classification in six bins; Col. 8: other spectra available from either SDSS or NED (SDSS-corr means that the SDSS spectrum is partly corrupted); Col. 9: independent classification of the nuclear activity from NED. 


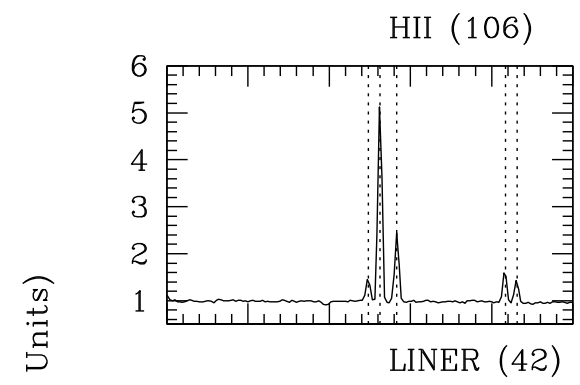

SEY $1(10)$
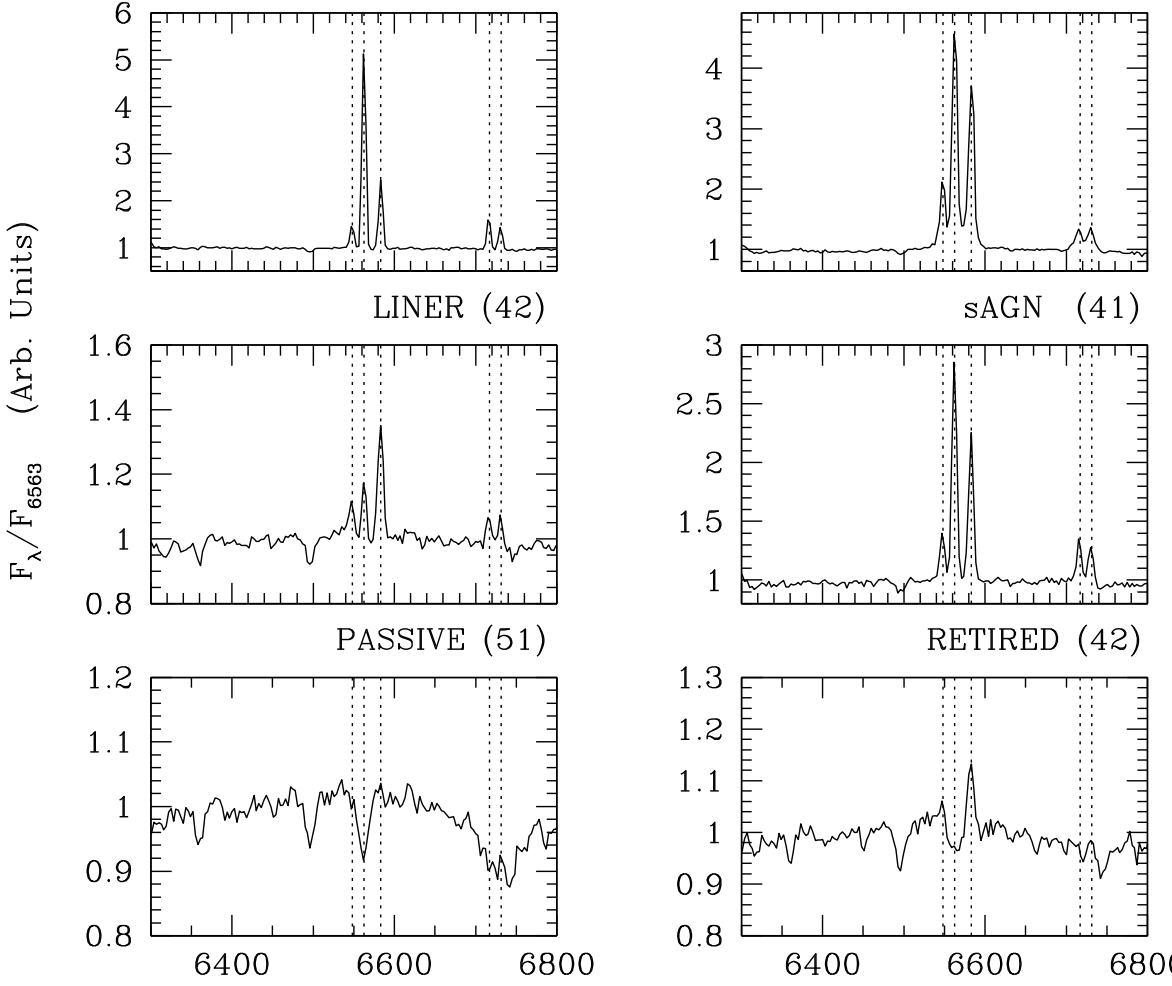
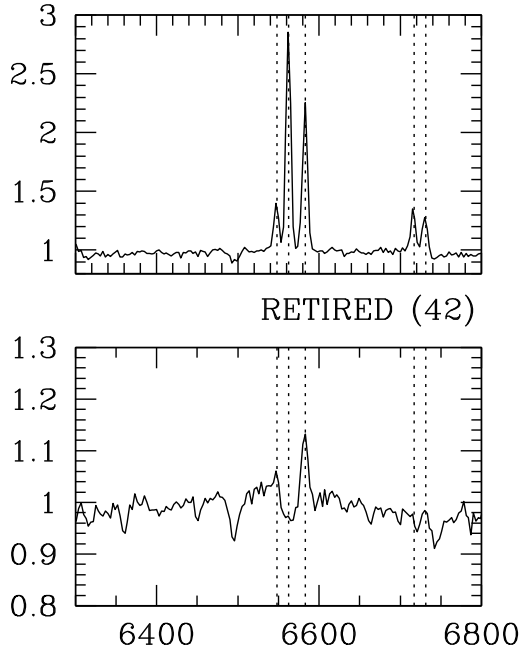

$\lambda_{\circ}(\AA)$

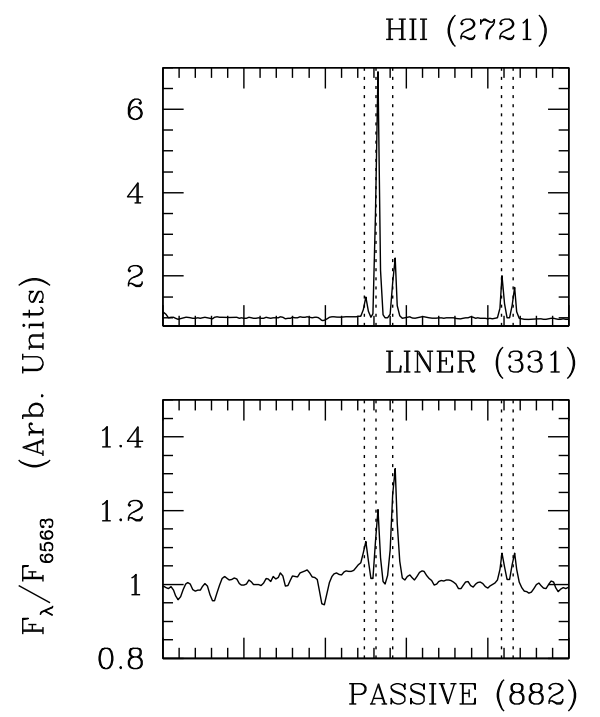

SEYFERT (48)
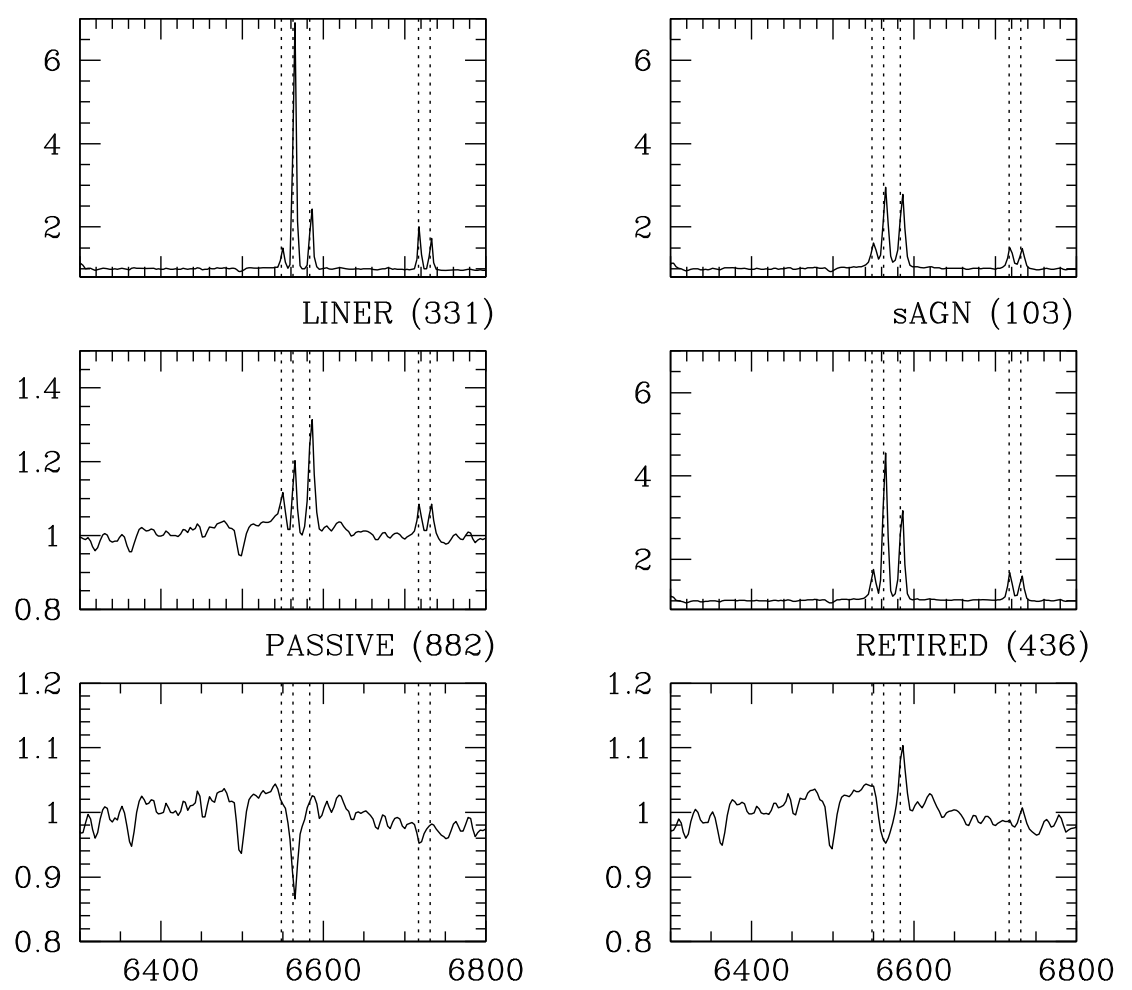

$\lambda_{\circ}(\AA)$

Fig. 4. Template spectra obtained by stacking individual normalized rest-frame spectra (whose number is given in parenthesis) in six bins of nuclear classification. The vertical dashed lines mark the position of [NII] $\lambda$ 6548, $\mathrm{H} \alpha \lambda 6563,[\mathrm{NII}]$ $\lambda 6584$, and the [SII] doublet $\lambda 6718$ and 16733. "LOI sample (top panel); "COMA sample" (bottom panel).

As anticipated in Sect. 2, the 283 "LOI sample" galaxies do not form a complete sample in any sense. Among them, 41 belong to the Virgo cluster, which are not considered further here. Not all of the 177 galaxies belonging to the Coma supercluster have new spectroscopic measurements: several have also a nuclear spectrum (acquired using three arcsec fibers) from the DR7 of SDSS and many others have nuclear spectra available from NED. Nevertheless, there are 130 Coma galaxies in the "LOI sample" (see Table 4) that do not have a spectrum in SDSS, hence these data contribute toward the completion of the 


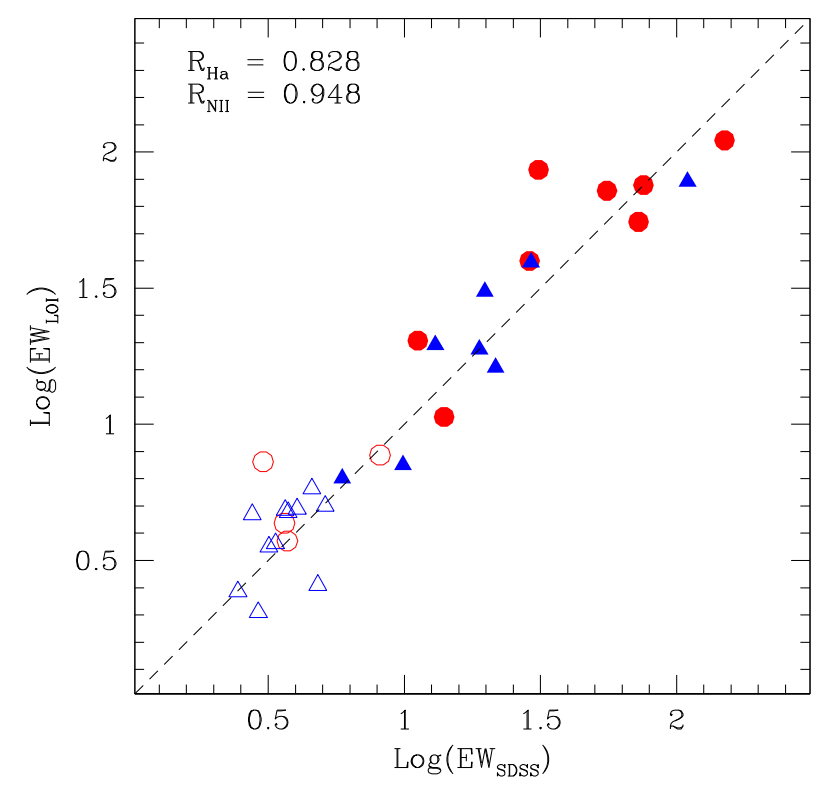

Fig. 5. The equivalent width of $\mathrm{H} \alpha$ (red dots) and $\lambda 6584$ [NII] (blue triangles) for 15 galaxies in the Coma supercluster that were measured both at Loiano and by SDSS. The correlation coefficients are given. The dashed line gives the 1:1 relation. Empty symbols refer to galaxies with luminosity $\log \left(L_{i} / L_{\odot}\right)>10.5$.

"COMA sample" (see Sect. 5). For 15 Coma galaxies taken at Loiano before the DR7 of SDSS was issued, Fig. 5 displays the two independent measurements of the EW of $\mathrm{H} \alpha$ and $\lambda 6584$ [NII], which do not appear to be affected by systematic errors ${ }^{6}$.

\section{AGNs in the Coma supercluster}

The data obtained in the previous section are used to help perform a census of AGNs in the Coma supercluster region.

Following an identical procedure to Gavazzi et al. (2010), the local number density $\rho$ around each galaxy was computed within a cylinder of $1 \mathrm{~h}^{-1} \mathrm{Mpc}$ radius and $1000 \mathrm{~km} \mathrm{~s}^{-1}$ half-length. We divide the sample into four overdensity bins, chosen in order to highlight physically different environments of increasing level of aggregation: the Ultra-Low density bin (UL: $\delta_{1,1000} \leq 0$ ) describing the underlying cosmic web; the Low density bin (L: $\left.0<\delta_{1,1000} \leq 4\right)$ comprising the filaments in the Great Wall and the loose groups; the High density bin (H: $\left.4<\delta_{1,1000} \leq 20\right)$ including the cluster outskirts and the significant groups; and the Ultra-High density bin (UH: $\left.\delta_{1,1000}>20\right)$ corresponding to the cores of rich clusters.

Figure 1 shows the celestial distribution and the wedge diagram for the 5027 galaxies in the "COMA sample", coded according to the four density bins.

Figure 6 (top panel) gives the $g-i$ color vs. $i$-band luminosity diagram for the studied galaxies, highlighting the distribution of galaxies without an SDSS spectrum and that the spectra taken at

\footnotetext{
6 The extraction of the Loiano spectra was carried out in apertures whose area $\left(11.6 \operatorname{arcsec}^{2}\right)$ is almost twice as large as that of the SDSS fibers $\left(7.1 \operatorname{arcsec}^{2}\right)$. This was done on purpose to compensate for the Loiano spectra being taken for the brightest galaxies, which are a factor of two, on average, larger than those taken by SDSS. Figure 5 shows no systematic effects for bright and faint objects.
}

Loiano (filled dots) were obtained deliberately for bright objects with $\log \left(L_{i} / L_{\odot}\right)>10$, where the missing spectra from SDSS are more abundant and AGNs are more frequently found (Decarli et al. 2007). Out of the 112 galaxies remaining with no spectra (empty circles), only 30 are brighter than $\log \left(L_{i} / L_{\odot}\right)=10$. Among them, we expect to miss approximately 10 AGNs, given the luminosity distribution of AGNs discussed below.

Before discussing the various color diagrams, we emphasize that the contribution of AGNs to the total $i$ luminosity is negligible, as the light measured in nuclear apertures of three arcsec diameter for the most luminous of all AGNs, i.e. the Seyferts, represents between $5 \%$ and $15 \%$ of the total light.

Figure 6 (middle panel) shows the position of HII, SEY, and sAGN in the color-magnitude diagram, clearly showing that AGNs of high activity belong to the high luminosity tail of the sequence composed of star-forming galaxies (their slope is 0.34 , and the dispersion is 0.20 ). Figure 6 (bottom panel) illustrates the position of PAS, RET, and LIN, showing that AGNs of low activity, including fake-AGNs, belong to the high luminosity part of the "red sequence" (their slope is 0.17 , and the dispersion is $0.10)$. LIN follow a relation with a slightly higher slope $(0.20)$ and dispersion (0.14) than RET and PAS alone (slope $=0.16$, and dispersion $=0.09$ ), suggesting that they might belong to a transition class between early- and late-type galaxies. From Fig. 6 (middle and bottom panels), we can see that many luminous objects, mostly AGNs, have redder colors than red-sequence galaxies of similar luminosity and in particular of PAS. These galaxies are strongly affected by extinction from dust lanes.

We have measured the average $g-i$ for the whole galaxy in the three classes of AGNs of decreasing activity (SEY, sAGN, LIN) and among RET. The resulting mean color for the four classes is $\langle g-i\rangle \simeq 1.15 \pm 0.15 \mathrm{mag}$, consistent with the average color of galaxies in the red sequence and of PAS galaxies. This value is not found to depend on the local galaxy density. The only class with $\langle g-i\rangle \simeq 0.72$, which is significantly bluer than all other classes, is the one of HII-like nuclei. Since $g-i$ color can be assumed as a proxy of the star formation in galaxies, this result seems to support the evolutionary scenario where the feedback from AGNs produces a significant quenching of the star formation in their host galaxies (e.g. Schawinski et al. 2009). However, our finding neither proves nor disproves this hypothesis because the same mean color might be found for PAS galaxies, which do not necessarly originate from a previous AGN phase, but simply from the fading of a nuclear starburst phase.

The morphology distribution of AGNs, including RET, is given separately in Fig. 7 (top panel). It appears that sAGN and SEY are mostly (40\%) associated with giant spiral galaxies (Sa$\mathrm{Sb}$ ) (and another $29 \%$ to S0a). LIN and RET are $78 \%$ associated with early-type galaxies (E-SOa).

Among non-AGNs (Fig. 7 bottom panel), PAS are $94 \%$ associated with E-S0, while HII regions are $87 \%$ associated with late-type galaxies (Sa-BCD).

The differential luminosity distribution of all AGNs (SEY+sAGN+LIN) (with and without RET) is given in Fig. 8. For all galaxies, the luminosity distribution follows a Schechter function (whose parameters can be found in Gavazzi et al. (2010). For the AGNs, the distribution is instead "Gaussian", with a peak near $\log \left(L_{i} / L_{\odot}\right)=10.2$. Below this luminosity $\left(\log \left(L_{i} / L_{\odot}\right) \leq 10.2\right)$, the fraction of Active Nuclei is $11 \%$ with RET and $6 \%$ without. For $\log \left(L_{i} / L_{\odot}\right)>10.2$, it becomes $60 \%$ with and $32 \%$ without RET. RET alone represent a similar fraction of all bona-fide AGNs at any luminosity.

When the frequency of galaxies in the various spectral classification bins (normalized to the total number of surveyed 


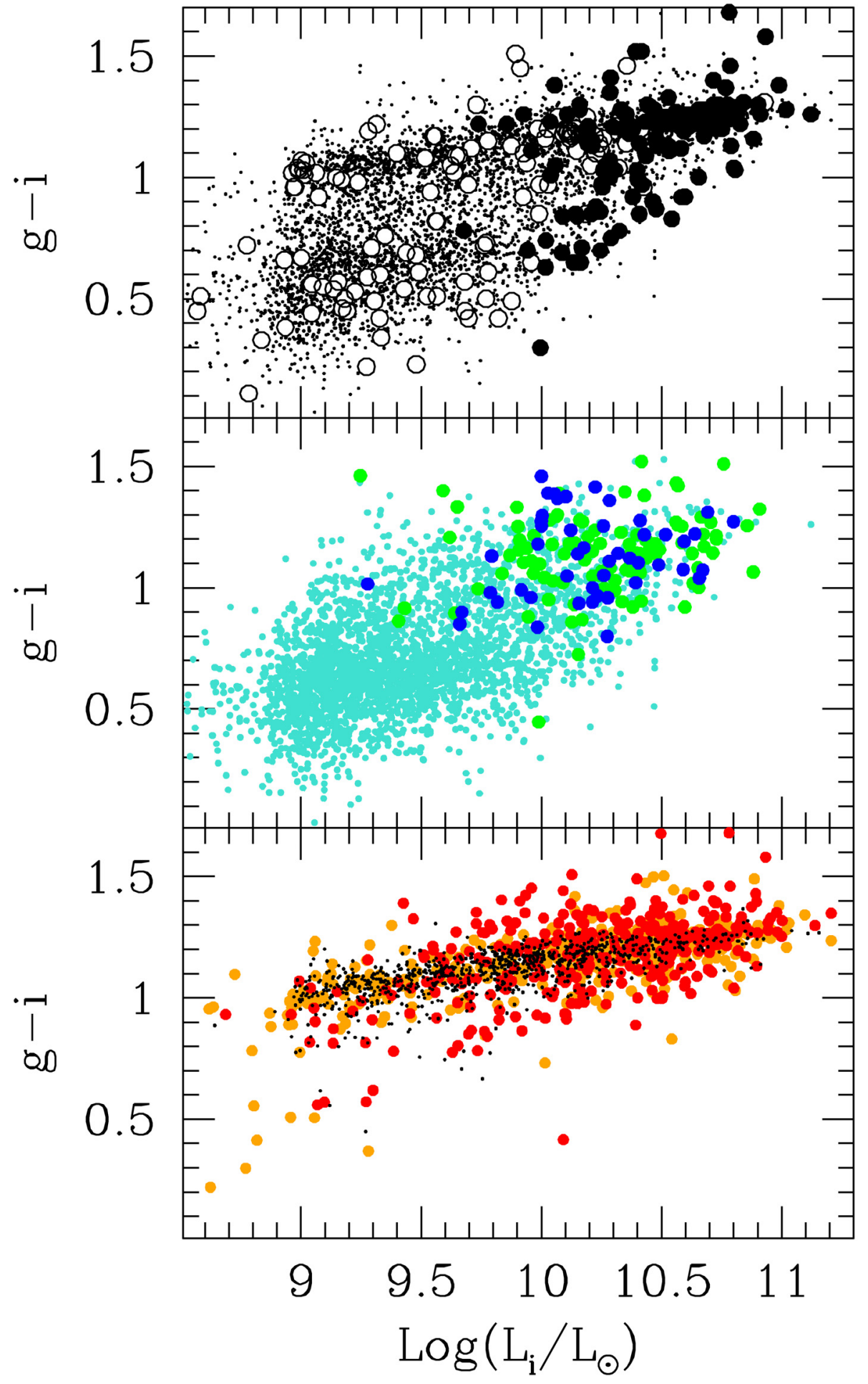

Fig. 6. The color $(g-i)$ vs. $i$-luminosity diagram of galaxies studied in this work. Top panel: small black dots represent the 4900 galaxies from SDSS; large filled symbols mark the 130 galaxies for which a nuclear spectrum was taken at Loiano, and large open symbols mark the 112 galaxies yet without spectra. Middle panel: HII regions (turquoise), SEY (blue), and sAGN (green) form the blue sequence. Bottom panel: LIN (red), RET (orange), and PAS (black) form the red sequence. galaxies) is plotted in bins of local galaxy density, it appears (see Fig. 9) that:

- among AGNs, the percentage of SEY-sAGN-LIN does not change significantly from UL to $\mathrm{H}$ density but decreases by a factor of two in the densest $\mathrm{UH}$ environment (notice that LIN are as frequent in the lowest and highest density bins);

- among non-AGNs, the fraction of HII-like nuclei decreases gradually with increasing density, overall by a factor of four. RET and PAS increase by a factor of two and four respectively. A similar result was obtained by Miller et al. (2003).

The second result need not imply that the local environment has a direct influence on the nuclear activity of galaxies, but partly reflects the influence of the environment on the "extended galaxy" that harbors some nuclear activity, also called "morphology segregation" (Dressler 1980). To distinguish the two effects, it is recommended that we normalize the number of objects in the various classes of nuclear activity not to the total number of surveyed galaxies (irrespective of the morphological type) but the number of galaxies of certain type classes, as in Fig. 10. Guided by the distribution of AGNs with respect to the Hubble classification (Fig. 7), it is apparent that (among AGNs) SEY and sAGN are more frequent in S0a-Sb, while LIN are frequent in E-Sbs. Among non-AGNs, HII are frequent in all late-type galaxies (including S0a and BCD), RET are found among earlytype galaxies (i.e. $\mathrm{dE}, \mathrm{dSO}, \mathrm{E}, \mathrm{S} 0+\mathrm{S} 0 \mathrm{a}$ ) and PAS in $\mathrm{dE}, \mathrm{dS} 0, \mathrm{E}$, 

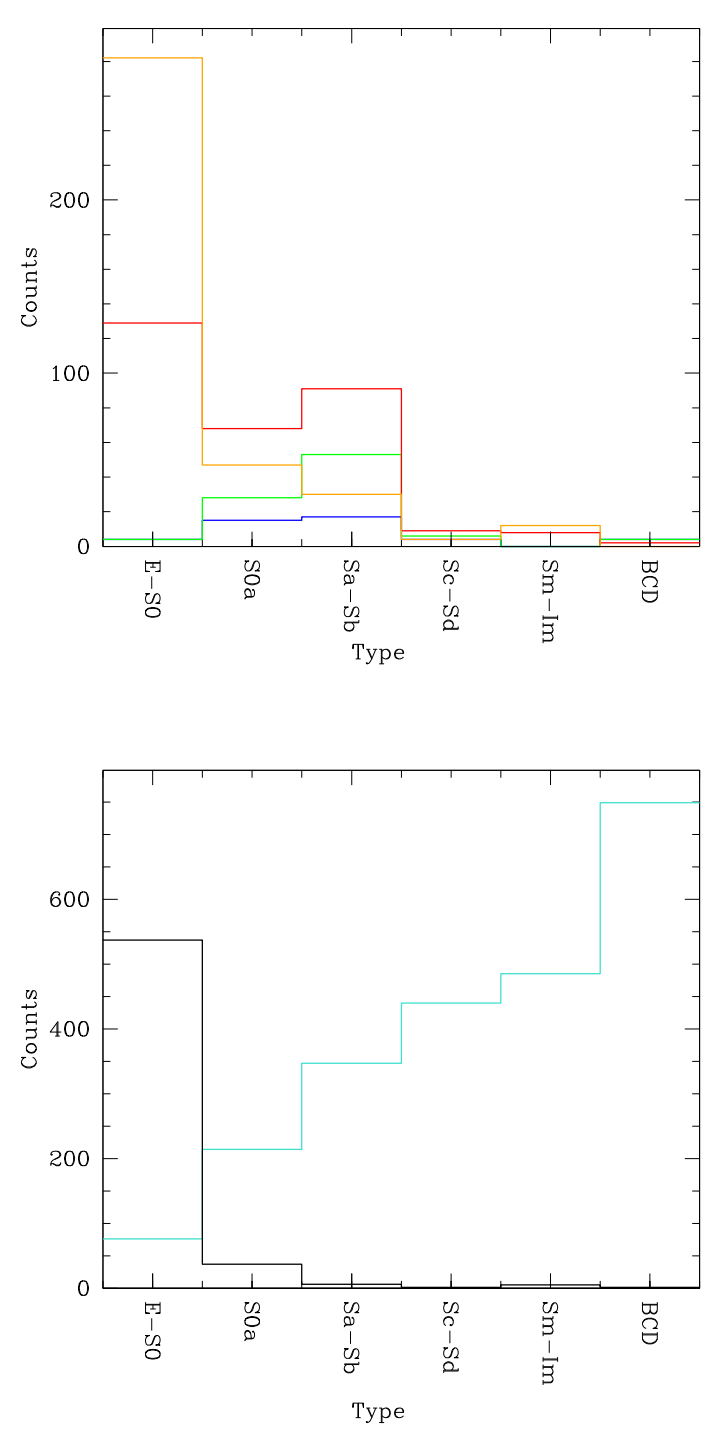

Fig. 7. The morphology distribution of: (top panel) SEY (Blue), LIN (red), sAGN (green), RET (orange); (bottom panel) HII (turquoise), PAS (black).

S0. Figure 10 allows us to study the nuclear activity of galaxies irrespective of "morphology segregation". In other words, Fig. 9 includes the effects of the environment on the galaxy as a whole (morphology) and on the nuclear region, while Fig. 10 emphasizes the effects of the environment on the nuclear region alone. In conclusion (see Fig. 10):

- the fraction of AGNs associated with both late- and earlytype giant galaxies is lower at most by a factor of two in the densest galaxy environment;

- the fraction of star-forming (HII) galaxies is lower by a factor of at most two in the densest galaxy environment;

- the fraction of ex-star-forming (RET) galaxies decreases gradually by a factor of two with increasing density from the lowest to the highest density bin;

- the fraction of passive (PAS) galaxies increases gradually by a factor of two with increasing density from the lowest to the highest density bin;

- all signs of nuclear activity, either associated with a central black hole or with star formation, are significantly lower

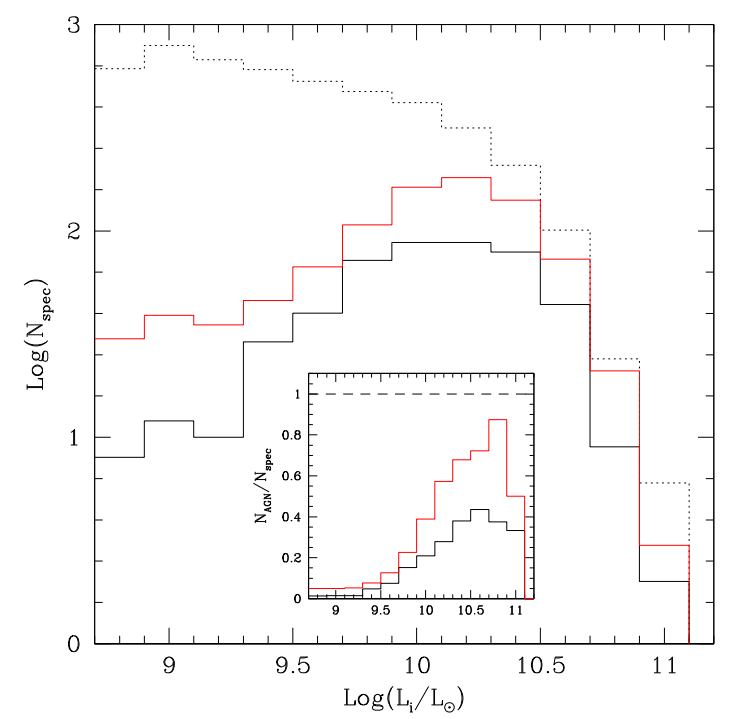

Fig. 8. The luminosity distribution of AGNs with (red) and without (solid black) RET and of all galaxies (dotted). The inset shows the ratio of AGNs to all galaxies with (red) and without (solid black) RET.

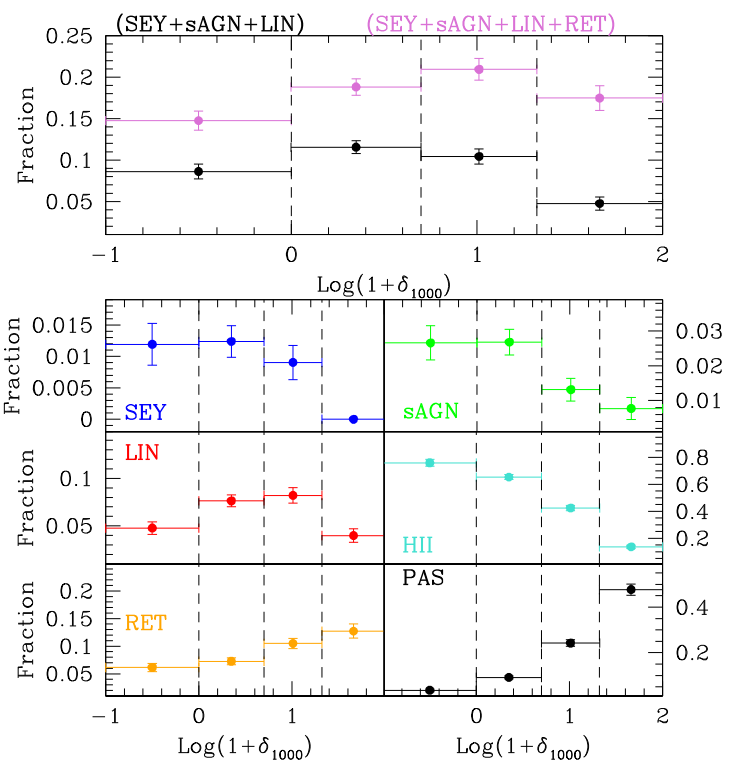

Fig. 9. The frequency distribution of SEY (top left); sAGN (top right); LIN (middle left), HII (middle right); RET (bottom left); PAS (bottom right) and all AGNs (top) with RET (pink) and without RET (black) in 4 bins of local galaxy density.

in the cores of the rich clusters than lower density environments.

\section{Discussion and conclusion}

A dependence of AGN activity on local galaxy density similar to the one found in this work was proposed by Mahajan et al. (2010), who analyzed almost exactly the same sky volume. This apparent agreement is however the consequence of a manifold blunder in their analysis. First of all, the galaxy sample in the Coma supercluster used by them suffers from $20 \%$ incompleteness at high luminosity (see Sect. 2) owing to the incompleteness 


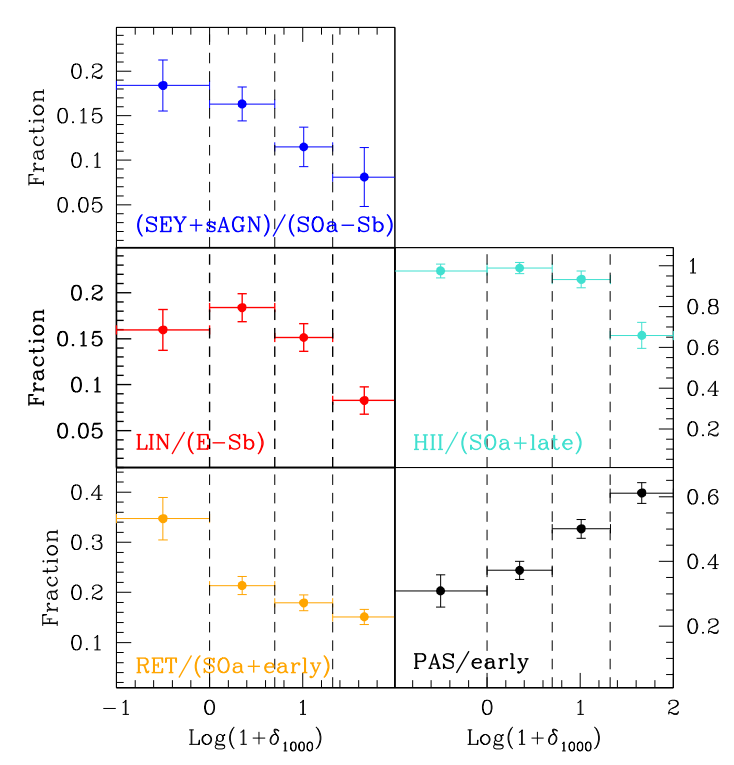

Fig. 10. The frequency distribution of the ratio of SEY+sAGN to giant spirals (SOa-Sb); of LIN to giant galaxies (E-Sb); of RET to earlytype galaxies (dE-SOa); of HII to SOa+late-type galaxies; and of PAS to early-type (dE-S0) galaxies in four bins of local galaxy density.

of the SDSS spectral database, which is entirely disregarded in the Mahajan et al. (2010) analysis.

Secondly the $\mathrm{H} \alpha$ spectral line measurements from the SDSS database have not been corrected for underlying stellar absorption. Thirdly the fraction of AGNs is computed by Mahajan et al. (2010) by normalizing to the total number of galaxies, irrespective of their morphology, Owing to the first omission and the adoption of the BPT diagnostic, Mahajan et al. (2010) failed to classify as AGNs an enormous number of objects with [NII] in emission and $\mathrm{H} \alpha$ in weak absorption, particularly ones present in the core of rich clusters, that would increase the frequency of AGNs in the densest environments. At the same time, they overestimated the number of AGNs among galaxies with $[\mathrm{NII}]$ and $\mathrm{H} \alpha$ in emission (that become HII like nuclei when corrected). These are predominantly found in the field. If Mahajan et al. (2010) had adopted the correct procedure in producing their BPT diagram, they would have concluded that the fraction of AGNs would increase, not decrease, with galaxy density. The reason why AGNs are missing in clusters is that most galaxies that are bona-fide AGNs according to the BPT diagnostic diagram become fake-AGNs (RET) according to the WHAN diagnostic diagram, making the whole issue of the influence of the environment on the formation of AGNs very slippery.

Using the AGN classification scheme of Kauffmann et al. (2003) based on their revised BPT diagram, Kauffmann et al. (2004) analyzed the properties of powerful AGNs among $\sim 122000$ galaxies with $14.5<r<17.77$ and median redshift $z \sim 0.1$, from the SDSS DR1. The subtraction of the underlying stellar continuum was performed by fitting the emission-linefree regions of the spectrum with a model galaxy spectrum. They concluded that powerful AGNs occur predominantly in massive galaxies with $>10^{10} M_{\odot}$, another manifestation of "downsizing" (Cowie et al. 1996; Gavazzi et al. 1996; Fontanot et al. 2009). Powerful AGNs are found among late-type, blue, star-forming galaxies. The decrease in their frequency with increasing galaxy density is mostly due to the decrease in the frequency of galaxies that are able to host AGNs (morphology segregation), but - to a lesser extent - is due to a genuine extra decrease in AGN frequency in high density environments. Von der Linden et al. (2010) argued that galaxies in dense environments are less likely to host a powerful optical AGN or a star-forming nuclear region. The frequency of optical AGNs in early-type galaxies declines in high density environments (by approximately a factor of two between the field and the center of clusters). They interpreted this finding by arguing that AGNs are fueled by mass loss from evolved stars. The mean age of the red galaxies increases towards the cluster center and provides less fuel for the central black hole.

Our analysis, which adopts the criteria of Cid Fernandes et al. $(2010,2011)$ for the classification of nuclei, is in full agreement with both Kauffmann et al. (2004) and Von der Linden et al. (2010). We conclude that, even after eliminating the effects of morphology segregation (by counting the frequency of nuclear activity in the proper bins of Hubble type), there is a residual decline in the frequency of nuclear activity (either triggered by black holes or sustained by the star formation) with increasing galaxy density (see Fig. 10). At any luminosity, this decline is quantified in a factor of $\sim 2$ per interval of density contrast of approximately 20, going from the less dense filaments that form the local cosmic web to the densest cores of rich clusters. Whichever mechanism is responsible for the suppression of over star formation in galaxies, and the migration of galaxy morphologies toward earlier Hubble types in rich and dense environments (see Boselli \& Gavazzi 2006; Blanton \& Moustakas 2009; Fumagalli \& Gavazzi 2008), it has far more severe consequences for their nuclear regions. We are not yet in the position to distinguish these two effects. However, we are in the process of obtaining a complete set of $\mathrm{H} \alpha$ imaging follow-up observations of the Coma supercluster that is appropriate for distinguishing the nuclear from the extended star-formation in galaxies in this representative part of the local Universe.

Acknowledgements. G. Gavazzi wishes to thank his first year master students who assisted during the observing nights at Loiano for many years. We also thank R. Gualandi and all night assistants of the Loiano Observatory, and the coordinator of the TAC, V. Zitelli for the generous time allocation. We thank O. Cucciati for computing the local galaxy density parameter. We are grateful to P. Franzetti and A. Donati for their contribution to GoldMine, the Galaxy On Line Database extensively used in this work (http://goldmine.mib.infn. it). We acknowledge the constructive criticism from an anonymous referee.

The present study could not have been conceived without the DR7 of SDSS Funding for the SDSS and SDSS-II has been provided by the Alfred P. Sloan Foundation, the Participating Institutions, the National Science Foundation, the US Department of Energy, the National Aeronautics and Space Administration, the Japanese Monbukagakusho, and the Max Planck Society, and the Higher Education Funding Council for England. The SDSS Web site is http://www . sdss.org/. The SDSS is managed by the Astrophysical Research Consortium (ARC) for the Participating Institutions. The Participating Institutions are the American Museum of Natural History, Astrophysical Institute Potsdam, University of Basel, University of Cambridge, Case Western Reserve University, The University of Chicago, Drexel University, Fermilab, the Institute for Advanced Study, the Japan Participation Group, The Johns Hopkins University, the Joint Institute for Nuclear Astrophysics, the Kavli Institute for Particle Astrophysics and Cosmology, the Korean Scientist Group, the Chinese Academy of Sciences (LAMOST), Los Alamos National Laboratory, the Max-PlanckInstitute for Astronomy (MPIA), the Max-Planck-Institute for Astrophysics (MPA), New Mexico State University, Ohio State University, University of Pittsburgh, University of Portsmouth, Princeton University, the United States Naval Observatory, and the University of Washington. G. Gavazzi acknowledges financial support from Italian MIUR PRIN contract 200854ECE5.

\section{References}

Abazajian, K. N., Adelman-McCarthy, J. K., Agüeros, M. A., et al. 2009, ApJS, 182,543

Baldwin, J. A., Phillips, M. M., \& Terlevich, R. 1981, PASP, 93, 5 
Binette, L., Magris, C. G., Stasińska, G., \& Bruzual, A. G. 1994, A\&A, 292, 13 Blanton, M. R., \& Moustakas, J. 2009, ARA\&A, 47, 159

Blanton, M. R., Schlegel, D. J., Strauss, M. A., et al. 2005a, AJ, 129, 2562

Blanton, M. R., Lupton, R. H., Schlegel, D. J., et al. 2005b, ApJ, 631, 208

Blanton, M. R., Eisenstein, D., Hogg, D. W., Schlegel, D. J., \& Brinkmann, J. 2005c, ApJ, 629, 143

Boselli, A., \& Gavazzi, G. 2006, PASP, 118, 517

Capetti, A., \& Baldi, R. D. 2011, A\&A, 529, A126

Cid Fernandes, R., Stasińska, G., Schlickmann, M. S., et al. 2010, MNRAS, 403, 1036

Cid Fernandes, R., Stasińska, G., Mateus, A., \& Vale Asari, N. 2011, MNRAS, 413, 1687

Cortese, L., Gavazzi, G., \& Boselli, A. 2008, MNRAS, 390, 1282

Cowie, L. L., Songaila, A., Hu, E. M., \& Cohen, J. G. 1996, AJ, 112, 839

Crane, P., Peletier, R., Baxter, D., et al. 1993, ApJ, 402, L37

Decarli, R., Gavazzi, G., Arosio, I. et al. 2007, MNRAS, 381, 136

Dressler, A. 1980, ApJ, 236, 351

Dressler, A., Thompson, I. B., \& Shectman, S. A. 1985, ApJ, 288, 481

Fontanot, F., De Lucia, G., Monaco, P., Somerville, R. S., \& Santini, P. 2009, MNRAS, 397, 1776

Fumagalli, M., \& Gavazzi, G. 2008, A\&A, 490, 571

Gavazzi, G., \& Perola, G. C. 1980, A\&A, 84, 228

Gavazzi, G., Perola, G. C., \& Jaffe, W. 1981, A\&A, 103, 35

Gavazzi, G., Pierini, D., \& Boselli, A. 1996, A\&A, 312, 397

Gavazzi, G., Boselli, A., Donati, A., Franzetti, P., \& Scodeggio, M. 2003, A\&A, 400,451
Gavazzi, G., Fumagalli, M., Cucciati, O., \& Boselli, A. 2010, A\&A, 517, A73 Giovanelli, R., Haynes, M. P., Kent, B. R., et al. 2005, AJ, 130, 2598 Hao, L., Strauss, M. A., Tremonti, C. A., et al. 2005, AJ, 129, 1783

Ho, L. C., Filippenko, A. V., \& Sargent, W. L. W. 1997, ApJS, 112, 315

Kauffmann, G., Heckman, T. M., Tremonti, C., et al. 2003, MNRAS, 346, 1055 Kauffmann, G., White, S. D. M., Heckman, T. M., et al. 2004, MNRAS, 353 713

Macchetto, F., Pastoriza, M., Caon, N., et al. 1996, A\&AS, 120, 463

Mahajan, S., Haines, C. P., \& Raychaudhury, S. 2010, MNRAS, 404, 1745

Miller, C. J., Nichol, R. C., Gómez, P. L., Hopkins, A. M., \& Bernardi, M. 2003, ApJ, 597, 142

Panessa, F., \& Bassani, L. 2002, A\&A, 394, 435

Popesso, P., \& Biviano, A. 2006, A\&A, 460, L23

Sarzi, M., Shields, J. C., Schawinski, K., et al. 2010, MNRAS, 402, 2187

Schawinski, K., Virani, S., Simmons, B., et al. 2009, ApJ, 692, L19

Shen, Y., Mulchaey, J. S., Raychaudhury, S., Rasmussen, J., \& Ponman, T. J. 2007, ApJ, 654, L115

Stasińska, G., Vale Asari, N., Cid Fernandes, R., et al. 2008, MNRAS, 391, L29

Strauss, M. A., Weinberg, D. H., Lupton, R. H., et al. 2002, AJ, 124, 1810

Trinchieri, G., \& di Serego Alighieri, S. 1991, AJ, 101, 1647

Véron-Cetty, M.-P., \& Véron, P. 2006, A\&A, 455, 773

von der Linden, A., Wild, V., Kauffmann, G., White, S. D. M., \& Weinmann, S. 2010, MNRAS, 404, 1231

Zwicky, F., Herzog, E., \& Wild, P. 1961-68, Pasadena: California Institute of Technology (CIT) 
Table 4. Parameters of 283 galaxies observed at Loiano.

\begin{tabular}{|c|c|c|c|c|c|c|c|c|}
\hline Gal & $\begin{array}{c}\text { RA } \\
\text { J2000 } \\
(2)\end{array}$ & $\begin{array}{c}\text { Dec } \\
\text { J20000 } \\
(3)\end{array}$ & (4) & $\begin{array}{c}\mathrm{EWH} \alpha \\
\AA \\
(5)\end{array}$ & $\begin{array}{c}\text { EW[NII2] } \\
\AA \\
(6)\end{array}$ & Class & Avsp. & Activity \\
\hline CGCG522-004 & $01: 47: 16.2$ & $+35: 33: 48$ & 0.01559 & -0.554 & -2.798 & LIN & - & - \\
\hline CGCG522-093 & $01: 58: 35.2$ & $+38: 43: 07$ & 0.01687 & -9.228 & -5.839 & sAGN & - & - \\
\hline CGCG522-104 & 02:00:59.7 & $+38: 47: 05$ & 0.01888 & -13.23 & -7.936 & sAGN & - & - \\
\hline CGCG522-106 & $02: 03: 44.8$ & $+38: 15: 31$ & 0.01922 & -3.166 & -2.952 & LIN & - & - \\
\hline NGC 0891 & $02: 22: 33.4$ & $+42: 20: 57$ & 0.00176 & -35.99 & -15.06 & HII & NED & - \\
\hline NGC 0925 & $02: 27: 16.9$ & $+33: 34: 45$ & 0.00184 & -35.87 & -8.241 & HII & NED & - \\
\hline UGC01935 & $02: 28: 14.5$ & $+31: 18: 42$ & 0.01665 & -144.8 & -10.65 & SY1 & - & Sey1 \\
\hline NGC 1068 & $02: 42: 40.7$ & $-00: 00: 48$ & 0.00379 & -81.52 & -150.9 & SY1 & NED & Sey2 \\
\hline UGC02855 & 03:48:20.7 & $+70: 07: 58$ & 0.00400 & -23.25 & -11.18 & HII & - & - \\
\hline NGC 1507 & $04: 04: 27.2$ & $-02: 11: 19$ & 0.00287 & -82.32 & -9.657 & HII & - & - \\
\hline NGC 1637 & $04: 41: 28.2$ & $-02: 51: 29$ & 0.00239 & -37.04 & -23.09 & sAGN & - & - \\
\hline NGC 1961 & $05: 42: 04.6$ & $+69: 22: 42$ & 0.01312 & -10.06 & -19.17 & sAGN & NED & LINER \\
\hline NGC 2273 & 06:50:08.7 & $+60: 50: 45$ & 0.00613 & -30.16 & -28.54 & SY1 & NED & Sey2 \\
\hline NGC 2339 & $07: 08: 20.5$ & $+18: 46: 49$ & 0.00735 & -37.87 & -28.26 & sAGN & NED & - \\
\hline UGC03809 & $07: 27: 04.1$ & $+80: 10: 41$ & 0.00735 & 0.910 & -1.431 & RET & NED & - \\
\hline NGC 2366 & $07: 28: 54.7$ & $+69: 12: 57$ & 0.00026 & -2339 & -20.87 & HII & NED & - \\
\hline NGC 2403 & $07: 36: 51.4$ & $+65: 36: 09$ & 0.00043 & -0.97 & -1.402 & LIN & NED & - \\
\hline NGC 2460 & $07: 56: 52.3$ & $+60: 20: 58$ & 0.00481 & -7.404 & -4.505 & sAGN & - & - \\
\hline NGC 2500 & 08:01:53.2 & $+50: 44: 14$ & 0.00168 & -1.484 & -0.099 & HII & NED & - \\
\hline NGC 2537 & $08: 13: 14.6$ & $+45: 59: 23$ & 0.00143 & -3.606 & -2.357 & HII & - & HII \\
\hline UGC04278 & 08:13:58.9 & $+45: 44: 32$ & 0.00180 & -129 & -10 & HII & SDSS & - \\
\hline CGCG119-016 & $08: 14: 14.2$ & $+21: 21: 20$ & 0.01129 & -0.319 & -2.227 & LIN & - & - \\
\hline CGCG119-029 & $08: 17: 25.8$ & $+21: 41: 08$ & 0.01189 & -9.690 & -5.93 & sAGN & - & - \\
\hline CGCG119-035 & $08: 17: 56.8$ & $+22: 26: 08$ & 0.00699 & -53.40 & -6.424 & HII & SDSS & - \\
\hline NGC 2549 & 08:18:58.4 & $+57: 48: 11$ & 0.00346 & 1.885 & -0.449 & PAS & NED & - \\
\hline CGCG119-055 & 08:19:38.0 & $+21: 06: 51$ & 0.01882 & -25.72 & -23.6 & sAGN & - & - \\
\hline CGCG119-056 & $08: 19: 41.3$ & $+22: 02: 31$ & 0.01151 & -48.29 & -9.248 & HII & SDSS & - \\
\hline CGCG119-057 & $08: 19: 48.3$ & $+22: 01: 53$ & 0.01194 & 1.481 & -1.812 & RET & - & - \\
\hline CGCG119-066 & $08: 20: 49.3$ & $+22: 39: 28$ & 0.01381 & -21.90 & -8.39 & HII & SDSS & - \\
\hline CGCG119-085 & $08: 24: 20.2$ & $+20: 32: 00$ & 0.01991 & -9.358 & -9.075 & sAGN & SDSS & - \\
\hline CGCG119-096 & $08: 26: 29.2$ & $+22: 15: 40$ & 0.02834 & -39.12 & -14.07 & HII & SDSS & - \\
\hline CGCG119-109 & $08: 27: 41.0$ & $+21: 28: 47$ & 0.01447 & -6.181 & -3.601 & HII & - & - \\
\hline NGC 2648 & $08: 42: 39.8$ & $+14: 17: 08$ & 0.00687 & 0.6825 & -2.224 & RET & - & - \\
\hline NGC 2654 & $08: 49: 11.9$ & $+60: 13: 16$ & 0.00449 & 0.0 & -1.235 & RET & SDSS & - \\
\hline NGC 2683 & $08: 52: 41.3$ & $+33: 25: 18$ & 0.00137 & -1.439 & -2.763 & LIN & NED & - \\
\hline NGC 2685 & $08: 55: 34.7$ & $+58: 44: 04$ & 0.00294 & 0.742 & -1.323 & RET & NED & Sey 2 \\
\hline UGC4881S & $09: 15: 54.0$ & $+44: 19: 49$ & 0.03948 & -36.35 & -19.07 & sAGN & - & - \\
\hline UGC4881N & 09:15:55.0 & $+44: 19: 58$ & 0.03974 & -24.8 & -15.72 & sAGN & - & - \\
\hline NGC 2841 & 09:22:02.6 & $+50: 58: 35$ & 0.00212 & -1.045 & -3.727 & LIN & NED & LINER \\
\hline NGC 2903 & 09:32:10.1 & $+21: 30: 03$ & 0.00185 & -52.78 & -19.8 & HII & NED & - \\
\hline NGC 3031 & $09: 55: 33.2$ & $+69: 03: 55$ & -0.0001 & -3.207 & -6.37 & LIN & NED & - \\
\hline NGC 3115 & $10: 05: 14.0$ & $-07: 43: 07$ & 0.00221 & 0.0 & 0.0 & PAS & NED & - \\
\hline CGCG123-035 & $10: 17: 39.7$ & $+22: 48: 36$ & 0.00391 & -10.12 & -4.748 & HII & - & - \\
\hline CGCG124-003 & $10: 20: 38.0$ & $+25: 30: 17$ & 0.02002 & 0.923 & 0.0 & PAS & - & - \\
\hline IC0610 & $10: 26: 28.4$ & $+20: 13: 41$ & 0.00390 & -6.495 & -2.79 & HII & - & - \\
\hline CGCG124-033 & 10:31:18.7 & $+25: 51: 13$ & 0.01950 & 3.353 & 0.0 & PAS & - & - \\
\hline NGC 3287 & $10: 34: 47.3$ & $+21: 38: 54$ & 0.00435 & -16.56 & -7.103 & HII & SDSS & - \\
\hline CGCG154-030 & $10: 36: 16.0$ & $+26: 57: 43$ & 0.02134 & 1.563 & 0.0 & PAS & - & - \\
\hline CGCG125-004 & $10: 45: 32.3$ & $+24: 09: 00$ & 0.02043 & -7.33 & -4.148 & HII & - & - \\
\hline CGCG155-023 & $10: 50: 22.7$ & $+26: 44: 06$ & 0.02168 & -11.81 & -3.975 & HII & - & - \\
\hline NGC 3412 & $10: 50: 53.3$ & $+13: 24: 44$ & 0.00280 & 1.67 & 0.0 & PAS & NED & - \\
\hline NGC 3424 & $10: 51: 46.3$ & $+32: 54: 03$ & 0.00498 & -19.56 & -13.84 & sAGN & SDSS & - \\
\hline NGC 3437 & $10: 52: 35.7$ & $+22: 56: 03$ & 0.00428 & -27.35 & -11.03 & HII & SDSS & - \\
\hline CGCG155-035 & $10: 54: 20.9$ & $+27: 14: 23$ & 0.00445 & -7.90 & -2.866 & HII & - & - \\
\hline NGC 3457 & $10: 54: 48.6$ & $+17: 37: 16$ & 0.00386 & 1.389 & -1.007 & RET & - & - \\
\hline $105825+241335$ & $10: 58: 25.2$ & $+24: 13: 35$ & 0.02145 & -1.527 & -3.268 & LIN & - & - \\
\hline NGC 3485 & 11:00:02.4 & $+14: 50: 30$ & 0.00479 & -10.05 & -5.731 & sAGN & - & - \\
\hline $110127+274310$ & $11: 01: 27.8$ & $+27: 43: 10$ & 0.02970 & 2.37 & -3.39 & RET & - & - \\
\hline CGCG267-037 & 11:03:11.0 & $+56: 13: 18$ & 0.00507 & 0.848 & -0.912 & RET & - & - \\
\hline NGC 3512 & 11:04:02.9 & $+28: 02: 13$ & 0.00459 & -1.319 & -2.938 & LIN & SDSS & - \\
\hline CGCG155-060 & $11: 05: 24.0$ & $+26: 21: 53$ & 0.02199 & 0.906 & -0.745 & RET & - & - \\
\hline CGCG125-036 & 11:09:54.5 & $+24: 15: 25$ & 0.02097 & -56.82 & -17.04 & HII & - & - \\
\hline NGC 3596 & $11: 15: 06.2$ & $+14: 47: 13$ & 0.00397 & -13.7 & -6.386 & HII & NED & - \\
\hline NGC 3599 & $11: 15: 27.0$ & $+18: 06: 37$ & 0.00277 & -0.315 & -1.2 & LIN & - & - \\
\hline
\end{tabular}


Table 4. continued.

\begin{tabular}{|c|c|c|c|c|c|c|c|c|}
\hline Gal & $\begin{array}{c}\text { RA } \\
\text { J2000 } \\
(2) \\
\end{array}$ & $\begin{array}{c}\text { Dec } \\
\text { J20000 } \\
(3) \\
\end{array}$ & $(4)$ & $\begin{array}{c}\text { EWH } \alpha \\
\AA \\
(5) \\
\end{array}$ & $\begin{array}{c}\text { EW[NII2] } \\
\AA \\
(6) \\
\end{array}$ & Class & $\begin{array}{l}\text { Avsp. } \\
\text { (8) }\end{array}$ & Activity \\
\hline CGCG096-038 & $11: 23: 45.5$ & $+17: 49: 07$ & 0.00428 & -30.55 & -9.221 & HII & - & - \\
\hline NGC 3657 & $11: 23: 55.6$ & $+52: 55: 16$ & 0.00405 & -4.844 & -2.347 & HII & - & - \\
\hline NGC 3683 & $11: 27: 31.8$ & $+56: 52: 37$ & 0.00572 & -9.177 & -3.803 & HII & SDSS & - \\
\hline CGCG126-070 & $11: 29: 51.0$ & $+24: 56: 16$ & 0.02515 & -2.233 & -1.356 & HII & - & - \\
\hline $113220+223748$ & $11: 32: 20.0$ & $+22: 37: 48$ & 0.03049 & -25.87 & -10.59 & HII & - & - \\
\hline $113507+201303$ & $11: 35: 07.0$ & $+20: 13: 03$ & 0.03119 & -15.68 & -10.2 & sAGN & - & - \\
\hline NGC 3758E & $11: 36: 29.0$ & $+21: 35: 45$ & 0.02850 & -186.9 & 12.08 & SY1 & - & - \\
\hline NGC 3758W & $11: 36: 29.0$ & $+21: 35: 45$ & 0.02948 & -22.31 & -13.09 & sAGN & - & - \\
\hline CGCG097-023 & $11: 36: 51.0$ & $+20: 00: 17$ & 0.02110 & 1.611 & 0.0 & PAS & - & - \\
\hline CGCG097-026 & $11: 36: 54.4$ & $+19: 58: 15$ & 0.02064 & -46.71 & -18.09 & HII & - & - \\
\hline CGCG127-006 & $11: 37: 43.0$ & $+22: 00: 34$ & 0.02982 & 1.574 & -1.428 & RET & - & - \\
\hline CGCG127-007 & $11: 37: 49.0$ & $+22: 01: 33$ & 0.03029 & 1.022 & -2.654 & RET & - & - \\
\hline CGCG127-012S & $11: 37: 53.7$ & $+21: 58: 51$ & 0.02911 & -1.472 & -2.095 & LIN & - & - \\
\hline NGC 3773 & $11: 38: 12.9$ & $+12: 06: 43$ & 0.00327 & -91.57 & -15.93 & HII & NED & Starb \\
\hline FGC1287 & $11: 39: 11.0$ & $+19: 35: 09$ & 0.02279 & -13.49 & -6.852 & HII & - & - \\
\hline CGCG097-044 & $11: 39: 47.5$ & $+19: 56: 00$ & 0.03646 & -5.509 & -6.402 & sAGN & - & - \\
\hline $113959+200935$ & $11: 39: 59.0$ & $+20: 09: 35$ & 0.02395 & 0.906 & 0.0 & PAS & - & - \\
\hline CGCG157-012 & $11: 40: 39.0$ & $+28: 51: 39$ & 0.02287 & -17.85 & -5.929 & HII & - & - \\
\hline CGCG127-025S & 11:40:44.2 & $+22: 25: 46$ & 0.02360 & -29.48 & -13.64 & HII & - & - \\
\hline CGCG127-025N & $11: 40: 44.6$ & $+22: 26: 49$ & 0.02353 & -39.82 & -19.58 & HII & SDSS & - \\
\hline CGCG127-032 & 11:42:09.1 & $+20: 18: 56$ & 0.01922 & 1.214 & -1.466 & RET & - & - \\
\hline UGC06678 & 11:43:01.9 & $+26: 15: 30$ & 0.03161 & 1.375 & -0.580 & RET & SDSS & - \\
\hline GCG097-082 & $11: 43: 24.6$ & $+19: 44: 59$ & 0.02034 & 2.273 & 0.0 & PAS & - & - \\
\hline CGCG097-092 & $11: 43: 58.2$ & $+20: 11: 06$ & 0.02125 & -77.24 & -21.96 & HII & - & - \\
\hline CGCG097-091 & $11: 43: 59.0$ & $+20: 04: 37$ & 0.02459 & -20.28 & -6.339 & HII & SDSS & - \\
\hline CGCG097-102N & $11: 44: 17.2$ & $+20: 13: 24$ & 0.02124 & 0.0 & 0.0 & PAS & - & - \\
\hline CGCG097-121 & $11: 44: 47.0$ & $+20: 07: 30$ & 0.02195 & -0.962 & -2.665 & LIN & - & - \\
\hline CGCG097-114 & $11: 44: 47.8$ & $+19: 46: 24$ & 0.02766 & -55.38 & -16.19 & HII & SDSS & - \\
\hline $114448+194828$ & $11: 44: 48.9$ & $+19: 48: 28$ & 0.06770 & -18.78 & -8.65 & HII & SDSS & - \\
\hline CGCG097-120 & $11: 44: 49.2$ & $+19: 47: 42$ & 0.01866 & -9.627 & -6.969 & sAGN & - & - \\
\hline CGCG097-122 & $11: 44: 52.2$ & $+19: 27: 15$ & 0.01823 & -31.86 & -11.2 & HII & - & - \\
\hline CGCG097-128 & $11: 45: 03.9$ & $+19: 37: 14$ & 0.02171 & 0.0 & 0.0 & PAS & - & - \\
\hline CGCG097-127 & $11: 45: 05.0$ & $+19: 36: 23$ & 0.02171 & -0.370 & -2.569 & LIN & SDSS & AGN \\
\hline CGCG097-129E & $11: 45: 07.0$ & $+19: 58: 01$ & 0.02517 & -5.977 & -6.482 & sAGN & - & - \\
\hline CGCG097-130 & $11: 45: 15.0$ & $-19: 23: 31$ & 0.02230 & 0.0 & 0.0 & PAS & - & - \\
\hline CGCG097-133E & $11: 45: 17.6$ & $+20: 01: 10$ & 0.04845 & -23.34 & -8.531 & HII & - & - \\
\hline CGCG097-134 & $11: 45: 30.0$ & $+19: 24: 00$ & 0.02483 & 1.966 & 0.0 & PAS & - & - \\
\hline CGCG097-139 & $11: 45: 49.5$ & $+19: 46: 03$ & 0.02320 & 0.0 & 0.0 & PAS & - & - \\
\hline CGCG127-051N & $11: 45: 59.4$ & $+20: 26: 49$ & 0.02370 & -26.34 & -11.14 & HII & - & - \\
\hline CGCG127-051S & $11: 45: 59.9$ & $+20: 26: 20$ & 0.02438 & -35.2 & -17.33 & HII & - & - \\
\hline CGCG127-054 & $11: 46: 47.2$ & $+20: 40: 32$ & 0.02343 & 0.0 & -2.258 & RET & SDSS & - \\
\hline $114658+194444$ & $11: 46: 58.0$ & $+19: 44: 44$ & 0.02073 & 1.345 & -1.358 & RET & - & - \\
\hline CGCG157-032 & 11:47:07.9 & $+29: 34: 39$ & 0.02271 & 0.0 & 0.0 & PAS & SDSS & - \\
\hline CGCG097-152 & $11: 47: 39.3$ & $+19: 56: 22$ & 0.02059 & 0.0 & 0.0 & PAS & SDSS & - \\
\hline CGCG157-035 & 11:48:03.4 & $+30: 21: 34$ & 0.02101 & -10.54 & -4.672 & HII & SDSS & - \\
\hline $114845+293828$ & $11: 48: 45.0$ & $+29: 38: 28$ & 0.02237 & -8.484 & -11.03 & SY1 & - & - \\
\hline CGCG127-063 & $11: 49: 59.3$ & $+21: 20: 01$ & 0.02624 & 0.5 & -0.73 & RET & - & - \\
\hline CGCG127-073 & $11: 51: 02.3$ & $+20: 47: 59$ & 0.02143 & 0.500 & -1.658 & RET & - & - \\
\hline CGCG127-075 & $11: 51: 21.0$ & $+21: 53: 21$ & 0.02648 & 0.0 & 0.0 & PAS & - & - \\
\hline CGCG127-076W & $11: 51: 26.6$ & $+22: 01: 41$ & 0.02834 & 1.401 & 0.0 & PAS & - & - \\
\hline CGCG127-076E & $11: 51: 28.2$ & $+22: 01: 33$ & 0.02566 & 0.0 & 0.0 & PAS & - & - \\
\hline CGCG157-045N & $11: 51: 32.1$ & $+27: 38: 49$ & 0.02891 & 0.0 & 0.0 & PAS & SDSS & - \\
\hline CGCG157-045S & $11: 51: 34.9$ & $+27: 38: 23$ & 0.02901 & 0.0 & 0.0 & PAS & - & - \\
\hline CGCG127-086 & $11: 52: 36.0$ & $+23: 34: 55$ & 0.02289 & 0.920 & 0.0 & PAS & - & - \\
\hline CGCG127-089 & $11: 52: 46.0$ & $+20: 59: 20$ & 0.02087 & 1.309 & 0.0 & PAS & - & - \\
\hline CGCG127-092 & 11:53:10.0 & $+20: 39: 28$ & 0.02333 & -1.583 & -6.848 & LIN & - & - \\
\hline CGCG127-094 & 11:53:17.0 & $+23: 27: 52$ & 0.02479 & -27.27 & -9.082 & HII & - & - \\
\hline $115348+250912$ & 11:53:48.1 & $+25: 09: 12$ & 0.02837 & -28.09 & -12.18 & HII & - & - \\
\hline CGCG127-101 & 11:53:57.5 & $+25: 41: 06$ & 0.01272 & 1.595 & 0.0 & PAS & - & - \\
\hline CGCG127-110 & $11: 57: 20.9$ & $+25: 11: 43$ & 0.01501 & -2.286 & -2.163 & LIN & - & - \\
\hline CGCG127-114W & $11: 57: 48.2$ & $+25: 16: 14$ & 0.01591 & -75.52 & -18.87 & HII & SDSS & - \\
\hline
\end{tabular}


Table 4. continued.

\begin{tabular}{|c|c|c|c|c|c|c|c|c|}
\hline (1) & $\begin{array}{c}\text { RA } \\
\text { J2000 } \\
(2)\end{array}$ & $\begin{array}{c}\text { Dec } \\
\text { J20000 } \\
(3)\end{array}$ & (4) & $\begin{array}{c}\mathrm{EWH} \alpha \\
\AA \\
(5) \\
\end{array}$ & $\begin{array}{c}\text { EW[NII2] } \\
\AA \\
(6) \\
\end{array}$ & Class & Av.sp. & Activity \\
\hline CGCG127-114E & $11: 57: 50.0$ & $+25: 16: 14$ & 0.01591 & -36.81 & -9.359 & HII & - & - \\
\hline CGCG157-064 & $11: 57: 51.0$ & $+29: 02: 20$ & 0.02156 & -41.94 & -14.21 & HII & - & - \\
\hline CGCG127-116 & $11: 57: 59.0$ & $+23: 12: 03$ & 0.02370 & 0.446 & -0.693 & RET & - & - \\
\hline CGCG157-065 & 11:58:05.2 & $+27: 52: 44$ & 0.01123 & -166.0 & -31.74 & HII & NED+SDSS & - \\
\hline CGCG157-067 & $11: 58: 20.7$ & $+28: 21: 35$ & 0.02827 & 0.0 & 0.0 & PAS & - & - \\
\hline CGCG127-122 & $11: 58: 42.9$ & $+25: 02: 25$ & 0.01457 & 0.7803 & -1.702 & RET & - & - \\
\hline CGCG127-130 & $11: 59: 36.0$ & $+21: 14: 49$ & 0.02635 & 0.136 & -1.189 & RET & - & - \\
\hline NGC 4031 & $12: 00: 31.0$ & $+31: 56: 51$ & 0.02607 & -21.34 & -9.448 & HII & - & - \\
\hline CGCG127-134 & $12: 01: 12.0$ & $+22: 37: 01$ & 0.02761 & -17.94 & -5.692 & HII & - & - \\
\hline CGCG013-046 & $12: 02: 42.2$ & $+01: 58: 37$ & 0.00659 & -13.95 & -9.624 & sAGN & NED+SDSS & - \\
\hline CGCG098-030 & $12: 02: 50.2$ & $+18: 00: 56$ & 0.01605 & -46.66 & -18.55 & HII & - & - \\
\hline CGCG128-003 & $12: 03: 27.0$ & $+22: 12: 36$ & 0.02160 & -27.95 & -9.976 & HII & - & - \\
\hline NGC 4067 & $12: 04: 11.5$ & $+10: 51: 16$ & 0.00805 & -0.269 & -1.121 & LIN & - & - \\
\hline CGCG098-046 & $12: 04: 32.5$ & $+20: 12: 18$ & 0.02072 & -0.974 & -4.754 & LIN & SDSS & - \\
\hline CGCG098-058 & $12: 07: 03.5$ & $+18: 31: 54$ & 0.02404 & 0.0 & -3.099 & RET & SDSS & - \\
\hline CGCG041-041 & $12: 07: 37.2$ & $+02: 41: 26$ & 0.00436 & -82.8 & -20.54 & HII & - & - \\
\hline CGCG041-042 & 12:08:11.1 & $+02: 52: 42$ & 0.00442 & -113.2 & -60.62 & sAGN & NED+SDSS & Starb \\
\hline CGCG128-038 & $12: 08: 15.3$ & $+25: 48: 41$ & 0.02234 & 0.0 & 0.0 & PAS & - & - \\
\hline CGCG098-074 & 12:09:58.0 & $+19: 55: 04$ & 0.02580 & -14.88 & -8.004 & HII & - & - \\
\hline CGCG158-036 & $12: 10: 18.3$ & $+26: 25: 51$ & 0.02174 & 0.0 & 0.0 & PAS & SDSS & - \\
\hline CGCG158-039 & $12: 11: 31.0$ & $+29: 05: 20$ & 0.02625 & 1.097 & 0.0 & PAS & - & - \\
\hline CGCG128-051 & $12: 11: 52.5$ & $+24: 07: 25$ & 0.00856 & -9.625 & -6.584 & sAGN & NED & - \\
\hline VCC0073 & $12: 13: 03.0$ & $+07: 02: 20$ & 0.00699 & -2.612 & -6.949 & LIN & - & - \\
\hline VCC0092 & $12: 13: 48.3$ & $+14: 54: 01$ & -0.0004 & -3.916 & -5.802 & LIN & NED & LINER \\
\hline VCC0097 & $12: 13: 53.6$ & $+13: 10: 22$ & 0.00827 & -14.82 & -8.86 & sAGN & SDSS & - \\
\hline VCC0099 & $12: 14: 02.2$ & $+06: 43: 24$ & 0.00815 & -5.344 & -2.127 & HII & SDSS & - \\
\hline VCC0120 & $12: 14: 38.6$ & $+05: 48: 21$ & 0.00688 & -29.58 & -9.817 & HII & - & - \\
\hline CGCG158-055 & $12: 15: 59.2$ & $+27: 26: 32$ & 0.02551 & -12.9 & -7.803 & sAGN & - & - \\
\hline VCC0199 & $12: 16: 33.8$ & $+07: 27: 44$ & 0.00868 & 0.0 & 0.0 & PAS & SDSS & - \\
\hline VCC0362 & $12: 19: 42.3$ & $+05: 32: 18$ & 0.00512 & -1.673 & -2.282 & LIN & - & - \\
\hline VCC0382 & $12: 19: 56.1$ & $+05: 20: 36$ & 0.00793 & -74.39 & -26.45 & HII & NED & - \\
\hline NGC 4287 & $12: 20: 48.5$ & $+05: 38: 24$ & 0.00718 & -22.1 & -8.184 & HII & - & - \\
\hline CGCG158-084 & $12: 21: 01.0$ & $+27: 53: 52$ & 0.02480 & 0.0 & 0.0 & PAS & - & - \\
\hline VCC0449 & $12: 21: 02.3$ & $+03: 43: 20$ & 0.00873 & -3.824 & -1.758 & HII & - & - \\
\hline VCC0492 & $12: 21: 41.5$ & $+05: 23: 05$ & 0.00770 & -3.436 & -3.098 & LIN & SDSS & - \\
\hline CGCG128-082 & $12: 21: 45.6$ & $+25: 53: 05$ & 0.02304 & 0.0 & -2.268 & RET & SDSS-corr & - \\
\hline VCC0562 & $12: 22: 35.9$ & $+12: 09: 29$ & 0.00014 & 0.0 & 0.0 & PAS & SDSS & - \\
\hline VCC0567 & $12: 22: 39.0$ & $+06: 40: 37$ & 0.00788 & -19.05 & -1.218 & HII & SDSS & - \\
\hline VCC0656 & $12: 23: 38.7$ & $+06: 57: 15$ & 0.00338 & -1.838 & -1.939 & LIN & SDSS & - \\
\hline VCC0697 & $12: 24: 05.5$ & $+07: 02: 29$ & 0.00410 & -15.52 & -5.061 & HII & SDSS & - \\
\hline VCC0792 & $12: 25: 22.2$ & $+10: 01: 01$ & 0.00321 & -1.133 & -1.827 & LIN & NED+SDSS & - \\
\hline CGCG158-106 & $12: 26: 19.9$ & $+26: 50: 18$ & 0.02361 & 0.0 & 0.0 & PAS & - & - \\
\hline VCC0912 & $12: 26: 32.2$ & $+12: 36: 40$ & 0.00034 & -45.58 & -14.27 & HII & SDSS & - \\
\hline VCC0958 & $12: 26: 56.4$ & $+15: 02: 51$ & -0.0008 & -3.428 & -5.434 & LIN & NED & - \\
\hline CGCG128-089 & $12: 26: 59.5$ & $+22: 38: 23$ & 0.02281 & 0.441 & -1.907 & RET & SDSS & - \\
\hline CGCG158-112 & $12: 27: 50.3$ & $+26: 59: 37$ & 0.02390 & -4.337 & -5.01 & LIN & SDSS & - \\
\hline CGCG159-002 & $12: 28: 15.4$ & $+28: 37: 13$ & 0.00220 & -8.183 & -4.646 & HII & NED & - \\
\hline VCC1110 & $12: 28: 29.6$ & $+17: 05: 06$ & 0.00651 & -0.413 & -3.252 & LIN & NED & LINER \\
\hline NGC 4460 & $12: 28: 45.6$ & $+44: 51: 51$ & 0.00163 & -34.77 & -7.781 & HII & NED & - \\
\hline VCC1145 & $12: 28: 59.0$ & $+03: 34: 14$ & 0.00294 & -0.965 & -3.249 & LIN & NED & - \\
\hline NGC 4490 & $12: 30: 36.2$ & $+41: 38: 38$ & 0.00188 & -10.55 & -2.92 & HII & NED & - \\
\hline VCC1330 & $12: 30: 59.7$ & $+08: 04: 40$ & 0.00580 & 1.616 & -1.427 & RET & - & - \\
\hline CGCG159-010 & $12: 31: 38.6$ & $+27: 29: 45$ & 0.02337 & -4.485 & -1.948 & HII & - & - \\
\hline VCC1412 & $12: 32: 06.2$ & $+11: 10: 35$ & 0.00447 & 2.314 & 0.0 & PAS & NED & - \\
\hline CGCG014-062 & $12: 32: 28.2$ & $+00: 23: 23$ & 0.00503 & -32.35 & 0.0 & HII & NED+SDSS & - \\
\hline VCC1442 & $12: 32: 36.5$ & $+02: 39: 41$ & 0.00576 & -78.15 & -9.636 & HII & - & - \\
\hline VCC1555 & $12: 34: 20.3$ & $+08: 11: 52$ & 0.00655 & -46.91 & -18.96 & HII & NED & - \\
\hline VCC1562 & $12: 34: 27.0$ & $+02: 11: 17$ & 0.00603 & -33.47 & -16.19 & HII & NED & - \\
\hline VCC1615 & $12: 35: 26.5$ & $+14: 29: 47$ & 0.00162 & 0.364 & -2.348 & RET & NED & LINER \\
\hline CGCG159-024 & $12: 35: 57.6$ & $+27: 57: 36$ & 0.00269 & -10.74 & -5.087 & HII & NED & - \\
\hline CGCG129-011 & $12: 36: 30.3$ & $+26: 11: 58$ & 0.02377 & 2.246 & -8.812 & RET & - & - \\
\hline VCC1690 & $12: 36: 49.8$ & $+13: 09: 47$ & -0.0007 & -5.7 & -8.776 & SY1 & NED & Sey \\
\hline CGCG159-033 & $12: 37: 21.1$ & $+28: 12: 29$ & 0.02559 & 0.0 & -4.089 & RET & SDSS & - \\
\hline
\end{tabular}


Table 4. continued.

\begin{tabular}{|c|c|c|c|c|c|c|c|c|}
\hline $\mathrm{Gal}$ & $\begin{array}{c}\text { RA } \\
\text { J2000 } \\
(2)\end{array}$ & $\begin{array}{c}\text { Dec } \\
\text { J20000 } \\
(3)\end{array}$ & (4) & $\begin{array}{c}\text { EWH } \alpha \\
\AA \\
(5)\end{array}$ & $\begin{array}{c}\text { EW[NII2] } \\
\AA \\
\text { (6) }\end{array}$ & Class & Av.sp. & Activity \\
\hline VCC1727 & $12: 37: 43.5$ & $+11: 49: 05$ & 0.00506 & -3.64 & -11.27 & SY1 & NED & Sey2 \\
\hline VCC1757 & $12: 38: 17.9$ & $+13: 06: 36$ & 0.00593 & -25.83 & -6.683 & HII & SDSS & - \\
\hline CGCG159-043 & $12: 39: 18.0$ & $+27: 46: 22$ & 0.02185 & 0.711 & -1.34 & RET & - & - \\
\hline CGCG159-045 & $12: 39: 18.0$ & $+28: 54: 17$ & 0.02490 & 1.626 & -2.058 & RET & - & - \\
\hline IC3644 & $12: 40: 36.2$ & $+26: 30: 17$ & 0.02212 & -13.98 & -4.167 & HII & - & - \\
\hline IC3646 & $12: 40: 38.5$ & $+26: 31: 34$ & 0.02159 & -38.46 & -11.31 & HII & - & - \\
\hline CGCG159-053 & $12: 41: 08.0$ & $+29: 32: 16$ & 0.02330 & 0.0 & -1.669 & RET & - & - \\
\hline NGC 4615 & $12: 41: 37.3$ & $+26: 04: 22$ & 0.01573 & -33.99 & -9.369 & HII & - & - \\
\hline NGC 4631 & $12: 42: 08.0$ & $+32: 32: 29$ & 0.00202 & -124.2 & -16.25 & HII & NED+SDSS & - \\
\hline VCC1923 & $12: 42: 31.1$ & $+03: 57: 37$ & 0.00245 & -251.9 & -70.92 & HII & - & - \\
\hline CGCG014-110 & $12: 42: 32.0$ & $-00: 04: 57$ & 0.00574 & -36.21 & -13.0 & HII & - & - \\
\hline CGCG129-020 & $12: 42: 54.0$ & $+20: 59: 23$ & 0.02182 & -0.316 & -2.462 & LIN & - & - \\
\hline CGCG100-005 & $12: 44: 53.1$ & $+18: 45: 19$ & 0.02205 & -29.25 & -16.64 & sAGN & - & - \\
\hline CGCG129-022 & $12: 45: 11.7$ & $+23: 02: 09$ & 0.02324 & -0.295 & -1.033 & LIN & - & - \\
\hline CGCG159-070 & $12: 45: 34.0$ & $+27: 03: 35$ & 0.02280 & 0.964 & -0.707 & RET & - & - \\
\hline CGCG159-071 & $12: 45: 43.0$ & $+29: 25: 58$ & 0.02315 & -34.99 & -11.54 & HII & - & - \\
\hline $124708+274736$ & $12: 47: 08.5$ & $+27: 47: 36$ & 0.02485 & -79.03 & -16.24 & HII & - & - \\
\hline UGC7955 & $12: 47: 11.0$ & $+26: 42: 48$ & 0.02236 & -8.625 & -2.832 & HII & - & - \\
\hline CGCG100-007 & $12: 47: 11.4$ & $+19: 27: 52$ & 0.02264 & 2.037 & -0.783 & RET & - & - \\
\hline VCC2070 & $12: 48: 22.9$ & $+08: 29: 15$ & 0.00336 & -0.47 & -2.432 & LIN & NED & Sey2 \\
\hline CGCG129-025 & $12: 49: 34.2$ & $+25: 28: 12$ & 0.01462 & -7.422 & -2.434 & HII & - & - \\
\hline $125209+272838$ & $12: 52: 09.0$ & $+27: 28: 38$ & 0.01949 & 2.212 & 0.0 & PAS & SDSS-corr & - \\
\hline CGCG071-068 & $12: 53: 50.8$ & $+09: 42: 36$ & 0.00944 & -56.73 & -23.17 & HII & SDSS & Starb \\
\hline CGCG160-008 & $12: 54: 19.0$ & $+27: 04: 05$ & 0.02790 & 0.0 & 0.0 & PAS & - & - \\
\hline CGCG043-068 & $12: 55: 23.6$ & $+07: 54: 34$ & 0.00934 & -18.77 & -6.371 & HII & - & - \\
\hline CGCG160-021 & $12: 56: 12.1$ & $+27: 44: 44$ & 0.02306 & 0.0 & 0.0 & PAS & - & - \\
\hline CGCG160-025 & $12: 56: 27.9$ & $+26: 59: 14$ & 0.02155 & -0.397 & -3.658 & LIN & SDSS & - \\
\hline CGCG160-055 & 12:58:05.6 & $+28: 14: 34$ & 0.02351 & -86.04 & -30.69 & HII & SDSS & - \\
\hline CGCG160-221 & $12: 59: 13.0$ & $+27: 58: 37$ & 0.02313 & 1.406 & 0.0 & PAS & - & - \\
\hline CGCG160-225 & $12: 59: 23.0$ & $+27: 54: 39$ & 0.02198 & 1.674 & 0.0 & PAS & - & - \\
\hline CGCG130-003 & $12: 59: 47.3$ & $+21: 48: 48$ & 0.02387 & -23.69 & -13.64 & sAGN & SDSS & - \\
\hline CGCG160-241 & 13:00:08.1 & $+27: 58: 37$ & 0.02166 & 1.099 & 0.0 & PAS & SDSS & - \\
\hline CGCG160-248 & 13:00:17.7 & $+27: 57: 19$ & 0.02302 & 0.0 & -1.286 & RET & - & - \\
\hline CGCG043-093 & 13:00:39.1 & $+02: 30: 05$ & 0.00320 & -62.39 & -20.17 & HII & NED+SDSS & - \\
\hline CGCG160-257 & $13: 00: 48.8$ & $+28: 09: 30$ & 0.01961 & -0.434 & -4.896 & LIN & SDSS & - \\
\hline CGCG160-258 & 13:00:51.0 & $+28: 02: 34$ & 0.02867 & 1.495 & 0.0 & PAS & - & - \\
\hline CGCG015-055 & 13:00:58.7 & $-00: 01: 39$ & 0.00396 & -64.74 & -20.66 & HII & SDSS & Starb \\
\hline CGCG160-261 & $13: 00: 59.3$ & $+27: 53: 59$ & 0.02300 & 2.189 & 0.0 & PAS & - & - \\
\hline CGCG160-096S & $13: 01: 24.5$ & $+29: 18: 30$ & 0.02386 & 1.254 & 0.0 & PAS & - & - \\
\hline CGCG160-096N & $13: 01: 25.3$ & $+29: 18: 50$ & 0.02316 & -110.3 & -77.82 & SY1 & SDSS & Sey2 \\
\hline CGCG160-095 & 13:01:26.1 & $+27: 53: 09$ & 0.01828 & -0.168 & -3.555 & RET & SDSS & - \\
\hline CGCG160-102 & $13: 01: 43.4$ & $+29: 02: 41$ & 0.02366 & -7.706 & -5.815 & sAGN & SDSS & - \\
\hline CGCG160-121 & 13:03:29.1 & $+26: 33: 02$ & 0.02226 & -3.732 & -2.039 & HII & SDSS & - \\
\hline CGCG130-005 & $13: 04: 10.8$ & $+22: 17: 23$ & 0.02386 & -72.05 & -39.34 & sAGN & SDSS & - \\
\hline $130553+273307$ & $13: 05: 53.1$ & $+27: 33: 08$ & 0.01888 & 1.296 & 0.0 & PAS & - & - \\
\hline CGCG160-154 & 13:10:20.0 & $+31: 26: 38$ & 0.02540 & 3.687 & -4.801 & RET & - & - \\
\hline CGCG160-163 & $13: 12: 59.4$ & $+27: 08: 30$ & 0.05972 & -18.42 & -13.12 & sAGN & - & - \\
\hline CGCG160-166 & $13: 13: 26.9$ & $+27: 48: 09$ & 0.02137 & -0.428 & -2.429 & LIN & SDSS & - \\
\hline CGCG130-021 & $13: 13: 45.3$ & $+24: 58: 57$ & 0.02418 & -34.63 & -15.94 & HII & - & - \\
\hline CGCG160-173 & $13: 16: 12.3$ & $+30: 57: 01$ & 0.01865 & -8.16 & -6.093 & sAGN & - & - \\
\hline CGCG160-177 & $13: 16: 51.0$ & $+31: 34: 52$ & 0.02980 & -10.94 & -5.381 & HII & - & - \\
\hline CGCG130-026 & $13: 17: 19.8$ & $+20: 38: 17$ & 0.02291 & -10.64 & -7.098 & sAGN & SDSS & - \\
\hline CGCG130-028 & $13: 17: 28.5$ & $+20: 36: 44$ & 0.02222 & 0.402 & -2.198 & RET & - & - \\
\hline CGCG160-181 & $13: 17: 30.6$ & $+31: 05: 34$ & 0.01851 & -15.01 & -5.528 & HII & - & - \\
\hline CGCG160-182 & $13: 17: 45.2$ & $+27: 34: 12$ & 0.02332 & -7.286 & -4.848 & sAGN & SDSS & - \\
\hline CGCG160-198 & 13:20:01.0 & $+31: 58: 53$ & 0.01813 & 0.0 & 0.0 & PAS & - & - \\
\hline CGCG160-200 & $13: 20: 14.0$ & $+30: 53: 59$ & 0.02448 & -34.14 & -15.18 & HII & - & - \\
\hline CGCG160-202 & $13: 20: 21.0$ & $+31: 30: 53$ & 0.01669 & -10.55 & -4.993 & HII & - & - \\
\hline NGC 5103 & 13:20:30.1 & $+43: 05: 02$ & 0.00425 & 1.232 & -0.522 & RET & - & - \\
\hline CGCG131-003 & $13: 21: 52.0$ & $+22: 25: 45$ & 0.03099 & 0.0 & -1.675 & RET & - & - \\
\hline CGCG131-004 & $13: 22: 26.3$ & $+21: 25: 33$ & 0.02284 & -42.45 & -25.62 & SY1 & - & - \\
\hline CGCG161-048 & $13: 25: 57.2$ & $+31: 37: 06$ & 0.02437 & 0.0 & 0.0 & PAS & SDSS & - \\
\hline
\end{tabular}


Table 4. continued.

\begin{tabular}{|c|c|c|c|c|c|c|c|c|}
\hline$\overline{\mathrm{Gal}}$ & $\begin{array}{c}\text { RA } \\
\text { J2000 } \\
(2)\end{array}$ & $\begin{array}{c}\text { Dec } \\
\text { J20000 } \\
(3)\end{array}$ & (4) & $\begin{array}{c}\text { EWH } \alpha \\
\AA \\
(5)\end{array}$ & $\begin{array}{c}\text { EW[NII2] } \\
\AA \\
(6)\end{array}$ & Class & Av.sp. & Activity \\
\hline CGCG016-069 & $13: 26: 19.7$ & $+02: 06: 03$ & 0.00362 & -4.201 & -2.502 & HII & NED & - \\
\hline CGCG161-073 & $13: 30: 26.0$ & $+31: 37: 15$ & 0.02441 & -0.306 & -2.201 & LIN & SDSS & - \\
\hline $135031+245834$ & $13: 50: 31.0$ & $+24: 58: 34$ & 0.03041 & 1.048 & -1.834 & RET & - & - \\
\hline CGCG132-023 & $13: 51: 05.4$ & $+25: 05: 37$ & 0.02923 & 0.0 & 0.0 & PAS & - & - \\
\hline $135244+250223$ & $13: 52: 44.0$ & $+25: 02: 23$ & 0.02857 & 1.11 & 0.0 & PAS & - & - \\
\hline NGC 5372 & $13: 54: 46.0$ & $+58: 40: 01$ & 0.00572 & -66.55 & -22.63 & HII & - & - \\
\hline CGCG132-047W & $13: 55: 28.4$ & $+25: 04: 25$ & 0.02955 & 0.0 & 0.0 & PAS & SDSS & - \\
\hline CGCG132-047C & $13: 55: 29.8$ & $+25: 04: 25$ & 0.02954 & 0.0 & 0.0 & PAS & - & - \\
\hline CGCG132-047E & $13: 55: 32.6$ & $+25: 04: 28$ & 0.02850 & 0.0 & 0.0 & PAS & - & - \\
\hline CGCG132-058 & $13: 57: 15.4$ & $+24: 15: 27$ & 0.02965 & -2.18 & -1.045 & HII & - & - \\
\hline IC4397 & $14: 17: 58.7$ & $+26: 24: 45$ & 0.01474 & -23.3 & -8.77 & HII & - & HII \\
\hline $141847+245625$ & $14: 18: 47.0$ & $+24: 56: 25$ & 0.01766 & -19.35 & -9.173 & HII & - & - \\
\hline CGCG047-022 & $14: 21: 13.1$ & $+03: 26: 09$ & 0.00496 & -6.435 & -3.329 & HII & - & - \\
\hline CGCG133-055 & $14: 26: 17.7$ & $+26: 14: 46$ & 0.03024 & -5.179 & -4.07 & sAGN & - & - \\
\hline CGCG163-050 & $14: 26: 59.6$ & $+26: 51: 50$ & 0.03015 & -10.65 & -3.947 & HII & - & - \\
\hline $142816+255055$ & $14: 28: 16.0$ & $+25: 50: 55$ & 0.01457 & 0.770 & 0.0 & RET & - & - \\
\hline CGCG163-058 & $14: 28: 31.8$ & $+27: 24: 32$ & 0.01439 & -3.236 & -5.218 & SY1 & - & - \\
\hline $143409+275649$ & $14: 34: 09.0$ & $+27: 56: 49$ & 0.03174 & 1.743 & -1.857 & RET & - & - \\
\hline CGCG133-088 & $14: 34: 12.7$ & $+25: 28: 05$ & 0.01614 & -11.72 & -6.65 & sAGN & - & - \\
\hline CGCG163-085 & $14: 35: 01.9$ & $+26: 32: 38$ & 0.03070 & -33.73 & -12.09 & HII & - & - \\
\hline CGCG134-016 & $14: 42: 18.0$ & $+25: 30: 34$ & 0.02662 & -3.055 & -3.797 & LIN & - & - \\
\hline UGC9489S & $14: 43: 01.0$ & $+25: 08: 48$ & 0.03083 & -69.68 & -10.7 & HII & - & - \\
\hline UGC9489N & $14: 43: 02.0$ & $+25: 09: 08$ & 0.03088 & -41.77 & -33.8 & sAGN & - & - \\
\hline CGCG164-031 & $14: 49: 27.0$ & $+27: 46: 51$ & 0.03097 & -0.877 & -2.039 & LIN & - & - \\
\hline $144954+274203$ & $14: 49: 54.3$ & $+27: 42: 03$ & 0.02973 & -30.69 & -9.636 & HII & - & - \\
\hline CGCG134-054 & $14: 54: 44.8$ & $+24: 05: 49$ & 0.01732 & -26.17 & -9.219 & HII & - & - \\
\hline CGCG134-066 & $15: 01: 53.7$ & $+25: 57: 52$ & 0.02205 & -2.708 & -5.947 & LIN & - & - \\
\hline IC4530 & $15: 03: 45.0$ & $+26: 06: 03$ & 0.03121 & -2.438 & -2.079 & LIN & - & - \\
\hline CGCG165-021 & 15:08:01.0 & $+27: 58: 20$ & 0.03196 & 0.767 & -1.769 & RET & - & - \\
\hline NGC 5957 & $15: 35: 23.2$ & $+12: 02: 51$ & 0.00609 & -1.279 & -3.044 & LIN & SDSS & - \\
\hline CGCG136-042 & $15: 39: 27.4$ & $+24: 56: 51$ & 0.02291 & -88.94 & -52.51 & sAGN & - & Starb \\
\hline CGCG136-067 & $15: 45: 16.0$ & $+24: 37: 50$ & 0.02233 & 1.482 & 0.0 & PAS & - & - \\
\hline NGC 6166 & $16: 28: 38.5$ & $+39: 33: 06$ & 0.03035 & -1.369 & -7.51 & LIN & - & - \\
\hline
\end{tabular}

